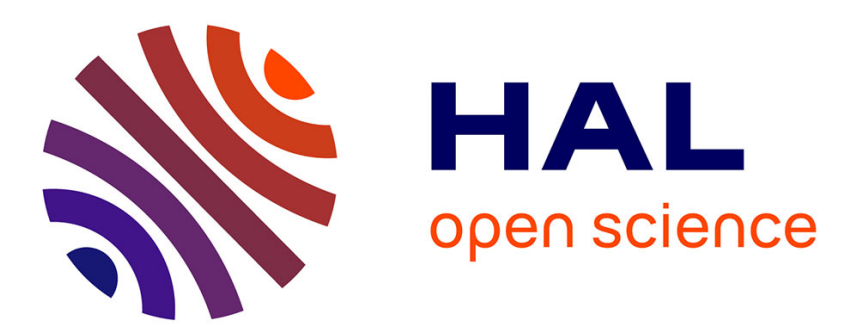

\title{
On the performance of the adaptive EWMA chart for monitoring time between events
}

Xuelong Hu, Philippe Castagliola, Jianlan Zhong, Anan Tang, Yulong Qiao

\section{To cite this version:}

Xuelong Hu, Philippe Castagliola, Jianlan Zhong, Anan Tang, Yulong Qiao. On the performance of the adaptive EWMA chart for monitoring time between events. Journal of Statistical Computation and Simulation, 2021, 91 (6), pp.1175-1211. 10.1080/00949655.2020.1843654 . hal-03191674

\section{HAL Id: hal-03191674 \\ https://hal.science/hal-03191674}

Submitted on 24 Aug 2021

HAL is a multi-disciplinary open access archive for the deposit and dissemination of scientific research documents, whether they are published or not. The documents may come from teaching and research institutions in France or abroad, or from public or private research centers.
L'archive ouverte pluridisciplinaire HAL, est destinée au dépôt et à la diffusion de documents scientifiques de niveau recherche, publiés ou non, émanant des établissements d'enseignement et de recherche français ou étrangers, des laboratoires publics ou privés. 


\title{
RESEARCH ARTICLE
}

\section{On the performance of the adaptive EWMA chart for monitoring time between events}

\author{
X.L. Hu ${ }^{\mathrm{a}}$ and P. Castagliola ${ }^{\mathrm{b}}$ and J.L. Zhong $^{\mathrm{c}}$ and A.A. Tang ${ }^{\mathrm{a}}$ and Y.L. Qiao ${ }^{\mathrm{d}}$

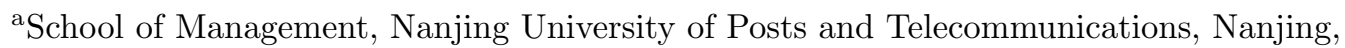 \\ China; ${ }^{\mathrm{b}} \mathrm{LS} 2 \mathrm{~N}$ UMR CNRS 6004 \& Université de Nantes, Nantes, France; ${ }^{\mathrm{c}}$ College of \\ Management and College of Tourism, Fujian Agriculture and Forestry University, Fuzhou, \\ China; ${ }^{\mathrm{d}}$ School of Automation, Nanjing University of Posts and Telecommunications, \\ Nanjing, China
}

\author{
ARTICLE HISTORY \\ Compiled August 24, 2021
}

\begin{abstract}
In high quality processes, events related to non-quality rarely occur and the times between these events (TBE) is likely to follow a skewed distribution like the gamma distribution. In order to monitor TBE data, an Adaptive Exponential Weighted Moving Average (AEWMA) control chart is proposed in this paper. This chart is designed to perform well over a range of shifts instead of being only efficient for a particular shift. An extensive performance analysis shows that, for most shifts, the Average Run Length (ARL) of the proposed chart performs better than other already existing control charts. In order to help the quality practitioner, some guidelines for choosing the most effective scheme in practice are provided. Finally, an illustrative example of the proposed scheme is also presented. Supplementary materials for this article are available online.
\end{abstract}

\section{KEYWORDS}

AEWMA chart; TBE; Gamma Distribution; Average Run Length

\section{Introduction}

As the most important tool in Statistical Process Monitoring (SPM), control charts are widely used for the monitoring of a process. Traditionally, control charts, such as the $\bar{X}, S^{2}$ and $R$ charts, are implemented for the monitoring of continuous characteristics in a process. For the monitoring of the number of non-conforming items in a process, the traditional approach is to use attribute charts, such as the $c$ or $u$ charts, see Montgomery [1]. Nowadays many processes produce a very small number of non-conforming items, like parts per million (ppm). These processes are usually called "high quality processes", where the occurrence of events (the occurrence of non-conforming items) are usually modelled as a homogeneous Poisson process. Instead of using the traditional attribute charts for monitoring the number of events, some researchers suggested to monitor the Time Between Events (TBE) which is, by definition, an exponential or a gamma distributed random variable in a homogeneous Poisson process (see Gan [2] and Zhang et al. [3]). TBE control charts have been 
applied in several areas, such as in the monitoring of the inter-failure time in a failure process (Xie et al. [4]), in health-care management (Xie et al. [5]), in earthquake occurrences (Santiago and Smith [6]) or in forest fires (Rahali et al. [7]).

Recently, TBE control charts have received much attention for the monitoring of high quality processes. For instance, Rakitzis [8] proposed a run rules Shewhart type chart for monitoring exponential distributed data. When process parameters are estimated, Kumar and Chakraborti [9] studied the conditional properties of a Phase II Shewhart chart for monitoring TBE type data. Kumar [10] investigated the properties of a Phase II exponential chart conditioned on a given Phase I sample, by deriving the exact expression of the distribution function of the conditional average run length $(C A R L)$. In addition, Xie et al. [11] pointed out that, although monitoring the failure occurrence process using an exponential TBE chart is straightforward, it may also causes many false alarms if the control limits are too wide and, therefore, they suggested to increase the sensitivity of such a control chart by monitoring the time between $r \geq 2$ failures, which is know to be a gamma distributed random variable. Following this, Zhang et al. [3] studied the performance of a Shewhart Gamma chart. Moreover, based on a class of absolutely continuous exponential distributions, Ali [12] evaluated the performance of TBE charts in terms of $A R L$, the expected quadratic loss, continuous ranked probability and the relative $A R L$. For other studies on Shewhart TBE charts, readers may also refer to Zhang et al. [13], Yang et al. [14], Ali and Pievatolo [15], Ali and Shah [16] and Ali et al. [17].

By only using the information in the current data, Shewart type charts are known to be able to detect large shifts quickly, while they are rather not sensitive to small shifts in processes. Apart from Shewhart type charts for TBE data, some studies on the Cumulative Sum (CUSUM) and exponentially weighted moving average (EWMA) charts for TBE data have also been conducted to improve the detection of small shifts. These charts are usually called memory type charts because they also use past samples information. Gan [2] first studied the properties of the one- and two-sided exponential EWMA charts. Khan et al. [18] proposed an EWMA control chart for monitoring exponential distributed data by transforming the sample data to approximate normal random variables and by constructing the EWMA statistic using the current and the previous moving average (MA) statistic for each subgroup. Liu et al. [19] investigated a CUSUM scheme for monitoring TBE data. The idea was also to transform the TBE data to approximate normal ones and then to apply the CUSUM scheme on these transformed data. The comparative studies showed that the proposed scheme had comparable performance with the exponential CUSUM chart. Qu et al. [20] proposed a weighted CUSUM chart with an additional power parameter to detect shifts in TBE. The results showed that the efficiency of the proposed chart can be actually improved by using this additional power parameter. For more research works on TBE control charts, readers can refer to the review conducted by Ali et al. $[21]$.

As pointed in Capizzi and Masarotto [22], a single EWMA chart cannot perform well for small and large shifts simultaneously in the process. To offer a more balanced detection against different shifts, they suggested an adaptive EWMA (AEWMA) chart to monitor process shifts. This chart can be seen as a smooth combination of a Shewhart chart and an EWMA chart. The results showed that this scheme gives a good protection against a wide range of shifts. From then on, several studies have 
been conducted on AEWMA type charts. Motivated by the work of Capizzi and Masarotto [22], Shu [23] extended the idea of the AEWMA chart for monitoring the process location to the case of monitoring the process dispersion. It has been shown that the AEWMA dispersion chart performs relatively better at both small and large changes of the process dispersion than the EWMA and other dispersion charts. Ugaz et al. [24] presented a family of adaptive control charts for monitoring the mean of a process by using a time-varying smoothing parameter. The proposed charts are efficient for a wide range of shifts, especially for small shifts. Considering measurement errors in the quality characteristic, Tang et al. [25, 26] studied the performance of the AEWMA mean and AEWMA median charts using a linearly covariate error model, respectively. When the process parameters are estimated from Phase I samples, the performance of the AEWMA chart was investigated in Saleh et al. [27] using a Markov chain approach. Moreover, considering the so-called between-practitioners variability in the estimated process parameters of the AEWMA chart, the standard deviation of $A R L$ combined with the average $A R L$ measures were used in Aly et al. [28] to re-evaluate the chart's performance. Tang et al. [29] introduced a new adaptive EWMA-type chart for monitoring the count data and they used a discrete-time Markov chain method to compute the exact run length properties of the proposed chart. Aly et al. [30] developed an AEWMA control chart for monitoring Zero Inflated Poisson (ZIP) processes and a Markov chain approach was used to obtain the properties of the proposed chart. The results showed that the ZIP AEWMA chart performed better than other competing charts for a wide range of process shifts. For more recent literatures on adaptive EWMA type charts, readers may also refer to Tang et al. [31], Tang et al. [32] and so on.

The literatures on the efficiency of EWMA type charts showed that they can only be constructed to perform well for either small or large shifts and, in order to circumvent this problem, AEWMA schemes have been proposed and they have been proven to show a good sensitivity for both small and large shifts simultaneously. The successful application of AEWMA charts motivates our investigation into the potential benefits of applying the AEWMA scheme to monitor TBE data. To the best of our knowledge, AEWMA TBE charts have not been studied in the literature so far. The purpose of this work is to fill this gap and to increase the sensitivity of TBE type control charts for both small and large shifts simultaneously.

As it has been shown in Acosta-Mejia and Pignatiello Jr. [33] for monitoring the process variance or in Xie et al. [4] for monitoring TBE data, a common problem with two-sided control charts for skewed distributions are their $A R L$-biased performance (i.e. some out-of-control $A R L$ values are larger than the predefined in-control $A R L$ value, see Morais et al. [34]). Since $A R L$-biased control charts cannot be effectively used for detecting all shifts in a process, to overcome this issue, one-sided charts for monitoring skewed distributions have been suggested, see Gan [2], Castagliola et al. [35], Zhang et al. [36] and so on. Thus in this paper, we consider two separate one-sided AEWMA charts for the detection of increasing and decreasing shifts in TBE data, respectively. Actually, if a two-sided AEWMA TBE chart is implemented for monitoring both increasing and decreasing shifts in the process, control limits of two-sided AEWMA TBE chart can be chosen following the algorithms in Tran and Knoth [37] to ensure its $A R L$-unbiased property.

In the subsequent section, control charts for Gamma distributed TBE data 
are introduced and the construction of two one-sided AEWMA TBE charts are presented using a Markov chain approach. Section 3 studies the design procedures and performance of the one-sided AEWMA TBE charts. Moreover, some comparisons are also made with the EWMA and Shewhart charts in this section. Then, a real data example is implemented to show the usage of the AEWMA TBE chart in Section 4. Finally, some conclusions and recommendations are made in Section 5.

\section{Control charts for Gamma distributed TBE data}

\subsection{Gamma distributed TBE data}

In a homogenous Poisson process, the inter-arrival time $Y_{i}, i=1,2, \ldots, r$, between two consecutive failures is an exponentially distributed random variable with the probability density function (p.d.f.) $f(y \mid \theta)=\frac{1}{\theta} e^{-\frac{y}{\theta}}$, where $\theta$ is the scale parameter (or mean) of the distribution. The sum $X=\sum_{i=1}^{r} Y_{i}$ of the these $r$ inter-arrival times between consecutive failures follows a $\operatorname{Gamma}(r, \theta)$ distribution with the following p.d.f.,

$$
f(x \mid r, \theta)=\frac{e^{-\frac{x}{\theta}} x^{r-1}}{\Gamma(r) \theta^{r}}, \quad x>0
$$

where the mean $E(X)$ and variance $V(X)$ of $X$ are $r \theta$ and $r \theta^{2}$, respectively. When $\theta=\theta_{0}$, the process is deemed to be in-control, where $\theta_{0}$ is the known in-control value of the scale parameter. Without loss of generality, $\theta_{0}=1$ is assumed in this paper. Otherwise, $\theta=\rho \theta_{0}(\rho \neq 1)$ means that the process is out-of-control. An increase (a decrease) in $\theta_{0}$ corresponds to $\rho>1(\rho<1)$, which is equivalent to an increase (a decrease) in the expected time between events. Following the work of Xie et al. [11], our aim is to construct control charts for monitoring changes in parameter $\theta$ of a Gamma distributed TBE random variable. If we are just interested in monitoring the time between two consecutive failures, the $\operatorname{Gamma}(1, \theta)$ distribution in Eq. (1) reduces to the exponential distribution, and the constructed control charts are for monitoring changes in $\theta$ of an exponential distributed TBE random variable. It should be pointed out that $\theta_{0}$ is assumed to be known here. If this parameter is not known in advance, it has to be estimated from a preliminary in-control samples. The unknown $\theta_{0}$ case will be studied in future investigations.

\subsection{The EWMA TBE chart for Gamma distributed data}

The standard EWMA chart uses a fixed weight $\lambda \in(0,1]$ to construct the monitoring statistic $Z_{t}=\lambda X_{t}+(1-\lambda) Z_{t-1}, t=1,2,3, \ldots$, where $X_{t}$ is the sample at time $t$ and $Z_{0}=r \theta_{0}$, and the upper $(U C L)$ and lower $(L C L)$ control limits of the EWMA TBE chart are determined by the constraint on the desired in-control performance. A process is considered to be in-control when $Z_{t} \in[L C L, U C L]$. Otherwise, when $Z_{t} \notin[L C L, U C L]$, the process is deemed to be out-of-control. It can be seen that when $\lambda=1$, the EWMA-TBE chart reduces to the Shewhart TBE chart.

Moreover, instead of using only a single two-sided EWMA chart for TBE data and to overcome the $A R L$-biased problem, Gan [2] suggested to implement two separate one-sided EWMA TBE charts for exponential distributed data. Similarly, for Gamma 
distributed TBE data, two one-sided EWMA charts are also defined as follows,

- First, an upper-sided EWMA chart for detecting an increase in the TBE data with the monitoring statistic,

$$
Z_{t}^{+}=\max \left(r \theta_{0}, \lambda X_{t}+(1-\lambda) Z_{t-1}^{+}\right),
$$

with the initial value $Z_{0}^{+}=r \theta_{0}$ and the upper control limit $U C L^{+}$.

- Second, a lower-sided EWMA chart for detecting a decrease in the TBE data with the monitoring statistic,

$$
Z_{t}^{-}=\min \left(r \theta_{0}, \lambda X_{t}+(1-\lambda) Z_{t-1}^{-}\right),
$$

with the initial value $Z_{0}^{-}=r \theta_{0}$ and the lower control limit $L C L^{-}$.

\subsection{Adaptive EWMA TBE chart for Gamma distributed data}

Because the EWMA chart cannot be designed to have a good sensitivity at both small and large shifts simultaneously, by adapting the weight $\lambda$ in the EWMA statistic as a function of the prediction error $e_{t}=X_{t}-Z_{t-1}$, Capizzi and Masarotto [22] suggested the AEWMA chart with the following monitoring statistic,

$$
Z_{t}=Z_{t-1}+\phi\left(e_{t}\right)=\omega\left(e_{t}\right) X_{t}+\left(1-\omega\left(e_{t}\right)\right) Z_{t-1},
$$

where $\omega\left(e_{t}\right)=\phi\left(e_{t}\right) / e_{t}$ is the weight of the AEWMA statistic and $\phi\left(e_{t}\right)$ is a score function. For the sake of simplicity, the score function $\phi\left(e_{t}\right)$ used here is limited to the Huber's score function, i.e.

$$
\phi(e)=\left\{\begin{array}{ll}
e+(1-\lambda) \times k & \text { if } e<-k \\
\lambda \times e & \text { if }|e| \leq k \\
e-(1-\lambda) \times k & \text { if } e>k
\end{array},\right.
$$

where $k \geq 0$. Note that when $k \rightarrow \infty, \phi(e) \approx \lambda e$ and the AEWMA chart converges to the EWMA chart. When $k \rightarrow 0, \phi(e) \approx e$ and the AEWMA chart converges to the Shewhart chart. If other score functions are used, similar methods can also be used to study the properties of the AEWMA chart.

Similar to the two separate one-sided EWMA charts for monitoring TBE data, two separate one-sided AEWMA charts are also suggested for monitoring the increase or decrease in TBE Gamma data. The monitoring statistics are, respectively, given by,

- First, an upper-sided AEWMA chart is suggested for detecting an increase in $\theta_{0}$

$$
Z_{t}^{+}=\max \left(r \theta_{0}, Z_{t-1}^{+}+\phi\left(e_{t}\right)\right)=\max \left(r \theta_{0}, \omega\left(e_{t}\right) X_{t}+\left(1-\omega\left(e_{t}\right)\right) Z_{t-1}^{+}\right),
$$

with the initial value $Z_{0}^{+}=r \theta_{0}$. An out-of-control signal is given when $Z_{t}^{+}$ exceeds the upper control limit $U C L^{+}$of the chart. 
- Second, a lower-sided EWMA chart is suggested for detecting a decrease in $\theta_{0}$,

$$
Z_{t}^{-}=\min \left(r \theta_{0}, Z_{t-1}^{-}+\phi\left(e_{t}\right)\right)=\min \left(r \theta_{0}, \omega\left(e_{t}\right) X_{t}+\left(1-\omega\left(e_{t}\right)\right) Z_{t-1}^{-}\right),
$$

with the initial value $Z_{0}^{-}=r \theta_{0}$. An out-of-control signal is given when $Z_{t}^{-}$is smaller than the lower control limit $L C L^{-}$of the chart.

If the aim is to detect both an increase and a decrease in TBE data, one can also use the combinations of these two one-sided AEWMA charts.

\subsection{A Markov chain method to compute the ARL of the proposed $A E W M A$ chart}

As one of the most widely used criterion, the $A R L$ is usually used to evaluate the performance of a control chart. The $A R L$ is defined as the expected number of charting statistics plotted on the chart until for the first time it gives an out-of-control signal. For fair comparisons, with the same in-control $A R L$ (hereafter denoted as $A R L_{0}$ ), the smaller the value of the out-of-control $A R L$ (hereafter denoted as $A R L_{1}$ ), the better the performance of the control chart. Other metrics, such as the median run length $(M R L)$ or other percentiles of the run length $(R L)$ values, can also be used to evaluate the performance of control charts, but this paper only focus on the $A R L$ metric to conform more with the most SPM literatures.

For the upper-sided AEWMA chart, a discretized Markov chain proposed in Lucas and Saccucci [38] is used here to approximate its $R L$ properties. This procedure involves dividing the interval $\left[r \theta_{0}, U C L^{+}\right]$into $p$ sub-intervals $\left(H_{j}-\Delta, H_{j}+\Delta\right], j=$ $1,2, \ldots, p-1, p$, each with width $2 \Delta$, where $\Delta=\frac{U C L^{+}-r \theta_{0}}{2 p}$. Similarly, $p$ sub-intervals of width $2 \Delta$, where $\Delta=\frac{r \theta_{0}-L C L^{-}}{2 p}$ can also be used for the lower-sided AEWMA chart. It can be seen that $H_{j}$ is the midpoint of the $j$ th subintervals and we define $H_{0}$ as the "restart state" of the chart. The regions above $U C L^{+}$(for the upper-sided chart) or below $L C L^{-}$(for the lower-sided chart) are considered as absorbing. When the number $p$ of sub-intervals is large enough (in this paper we choose $p=200$ ), this approach can effectively evaluate the $R L$ properties of the chart. Then the probability $Q_{i, j}$ that the control statistic $Z_{t}^{+}\left(Z_{t}^{-}\right)$moves from state $i$ to state $j$ in one step is given as follows,

- For the upper-sided AEWMA chart,

$$
Q_{i, j}= \begin{cases}P\left(Z_{t}^{+} \leq r \theta_{0} \mid Z_{t-1}^{+}=H_{i}\right), & \text { if } j=0, \\ P\left(H_{j}-\Delta<Z_{t}^{+} \leq H_{j}+\Delta \mid Z_{t-1}^{+}=H_{i}\right), & \text { if } j=1,2, \cdots, p\end{cases}
$$

- For the lower-sided AEWMA chart,

$$
Q_{i, j}= \begin{cases}P\left(Z_{t}^{-}>r \theta_{0} \mid Z_{t-1}^{-}=H_{i}\right), & \text { if } j=0, \\ P\left(H_{j}-\Delta<Z_{t}^{-} \leq H_{j}+\Delta \mid Z_{t-1}^{-}=H_{i}\right), & \text { if } j=1,2, \cdots, p\end{cases}
$$


Using the score function $\phi(e)$ in Equation (3), the inverse function $\phi^{-1}(z)$ is,

$$
\phi^{-1}(z)= \begin{cases}z-(1-\lambda) \times k & \text { if } z<-\lambda k \\ z / \lambda & \text { if }|z| \leq \lambda k \\ z+(1-\lambda) \times k & \text { if } z>\lambda k\end{cases}
$$

After some manipulations, the transition probability $Q_{i, j}$ is given as follows:

- If $j=0$ (for the upper-sided AEWMA chart),

$$
\begin{aligned}
Q_{i, 0} & =P\left(X_{t} \leq \phi^{-1}\left(r \theta_{0}-H_{i}\right)+H_{i}\right), \\
& =F\left(\phi^{-1}\left(r \theta_{0}-H_{i}\right)+H_{i}\right),
\end{aligned}
$$

- If $j=0$ (for the lower-sided AEWMA chart),

$$
\begin{aligned}
Q_{i, 0} & =1-P\left(X_{t}<\phi^{-1}\left(r \theta_{0}-H_{i}\right)+H_{i}\right), \\
& =1-F\left(\phi^{-1}\left(r \theta_{0}-H_{i}\right)+H_{i}\right),
\end{aligned}
$$

- If $j=1,2, \cdots, p$

$$
\begin{aligned}
Q_{i, j} & =P\left(H_{i}+\phi^{-1}\left(H_{j}-H_{i}-\Delta\right)<X_{t} \leq H_{i}+\phi^{-1}\left(H_{j}-H_{i}+\Delta\right)\right), \\
& =F\left(H_{i}+\phi^{-1}\left(H_{j}-H_{i}+\Delta\right)\right)-F\left(H_{i}+\phi^{-1}\left(H_{j}-H_{i}-\Delta\right)\right),
\end{aligned}
$$

where $F(x \mid r, \theta)=\frac{1}{\theta^{r} \Gamma(r)} \int_{0}^{x} t^{r-1} e^{-\frac{t}{\theta}} \mathrm{d} t$ is the cumulative distribution function (c.d.f.) of $X$.

Then the transition probability matrix $\mathbf{Q}$ with probabilities $Q_{i, j}$ corresponding to the $p+1$ transient states is,

$$
\mathbf{Q}=\left(\begin{array}{ccccc}
Q_{0,0} & Q_{0,1} & \cdots & Q_{0, p-1} & Q_{0, p} \\
Q_{1,0} & Q_{1,1} & \cdots & Q_{1, p-1} & Q_{1, p} \\
\vdots & \vdots & & \vdots & \vdots \\
Q_{p-1,0} & Q_{p-1,1} & \cdots & Q_{p-1, p-1} & Q_{p-1, p} \\
Q_{p, 0} & Q_{p, 1} & \cdots & Q_{p, p-1} & Q_{p, p}
\end{array}\right)
$$

The $R L$ distribution of the proposed AEWMA TBE chart can be obtained by the transition probability matrix $\mathbf{Q}$ and the initial probability vector $\mathbf{q}=(1,0, \ldots, 0)$, i.e., the initial state is assumed to be the "restart state". Since the $(R L)$ of the AEWMA TBE chart is a Discrete Phase-type $(\mathrm{DPH})$ random variable of parameters $(\mathbf{Q}, \mathbf{q})$, the p.d.f. $f_{R L}(\ell \mid \mathbf{Q}, \mathbf{q})$ and the c.d.f. $F_{R L}(\ell \mid \mathbf{Q}, \mathbf{q})$ of the $R L$ distribution of the AEWMA TBE chart is (see Neuts [39] and Latouche and Ramaswami [40]),

$$
\begin{aligned}
f_{R L}(\ell \mid \mathbf{Q}, \mathbf{q}) & =\mathbf{q}^{\top} \mathbf{Q}^{\ell-1}(\mathbf{I}-\mathbf{Q}) \mathbf{1} \\
F_{R L}(\ell \mid \mathbf{Q}, \mathbf{q}) & =1-\mathbf{q}^{\top} \mathbf{Q}^{\ell} \mathbf{1}
\end{aligned}
$$

where $\mathbf{I}$ is the $(p+1, p+1)$ identity matrix and $\mathbf{1}$ is a $(p+1,1)$ vector of 1 's. Moreover, 
the expected time to absorption of the Markov chain is $A R L=E(R L)$,

$$
A R L=\mathbf{q}^{\boldsymbol{\top}}(\mathbf{I}-\mathbf{Q})^{-1} \mathbf{1} .
$$

\section{Performance of the proposed one-sided AEWMA TBE charts}

In order to investigate the performance of the proposed one-sided AEWMA TBE Gamma charts, the chart parameters $\left(\lambda, k, U C L^{+}\right)$or $\left(\lambda, k, L C L^{-}\right)$have to be selected first. Following the works in Shu [23] and $\mathrm{Su}$ et al. [41], the joint effect of $\lambda$ and $k$ on the $A R L$ performance of the AEWMA chart is first investigated to provide some insight into the choice of the design parameters. Then, the nearly "optimal" design parameters of the AEWMA chart for both a small shift $\rho_{1}$ and a large shift $\rho_{2}$ in TBE data are obtained using a sequential searching procedure providing a good overall performance of the chart.

\subsection{The joint effect of parameters $\lambda$ and $k$}

Following the works in Shu [23] and $\mathrm{Su}$ et al. [41], several contour plots of $A R L_{1}$ values for different shifts of the upper-sided AEWMA TBE chart as a function of parameters $(\lambda, k)$ are presented in Figure 1 when the desired $A R L_{0}=200$ and $r=2$.

\section{(Please Insert Figure 1 Here)}

It can be concluded from Figure 1 that, when the purpose is to detect a relative small increase in the process, for instance, when $\rho=1.2$ in (a) and $\rho=1.5$ in (b), the $A R L_{1}$ value tends to increase as $\lambda$ increases or as $k$ decreases. The suggested values of $\lambda$ and $k$ for detecting small increasing shifts in the process are smaller than 0.2 and are within the range $[5.5,7]$, respectively. On the other hand, if the purpose is to detect a relative large increase in the process, for instance, when $\rho=5$ in (d), the $A R L_{1}$ value decreases as $\lambda$ increases or $k$ decreases. The suggested values of $\lambda$ and $k$ for detecting large increasing shifts in the process are roughly within the ranges $[0.2,0.7]$ and $[3.5,5.5]$, respectively. Similar conclusions of the trends in $A R L$ as a function of $\lambda$ and $k$ also hold for other cases of $r=1$ (exponential) or $r>2$. For simplicity, these contour plots are not presented here.

As the counterpart of the upper-sided AEWMA TBE chart, the $A R L_{1}$ of the lower-sided AEWMA TBE chart also shows similar performance as $\lambda$ and $k$ change, i.e. if the aim is to detect a small decreasing shift in the process, the $A R L_{1}$ value tends to increase as $\lambda$ increases or as $k$ decreases. On the other hand, if the aim is to detect a large decreasing shift in the process, the $A R L_{1}$ value tends to decrease as $\lambda$ increases or $k$ decreases. For simplicity, all the contour plots of the $A R L_{1}$ values for different shifts of the lower-sided AEWMA chart as a function of parameters $(\lambda, k)$ are not presented here. 


\subsection{Design procedures of the AEWMA TBE chart}

Because the two-stages procedure proposed in Capizzi and Masarotto [22] to search the optimal parameters of the AEWMA chart is complicated and requires extensive computations, Shu [23] proposed a sequential searching procedure to find the "nearly" optimal parameters of the AEWMA type chart. This approach is simple and requires less computation work. In this paper, we also adopt this sequential searching approach to the AEWMA TBE chart. Details of the procedure are summarized as follows,

(1) Choose a desired in-control value $A R L_{0}$, the parameter $r$ of the Gamma distribution, a small shift $\rho_{1}$ and a large shift $\rho_{2}$.

(2) Find the optimal value of the smoothing parameter $\lambda$ of the one-sided EWMA TBE charts for the specified small shift $\rho_{1}$ with the constraint on the desired $A R L_{0}$.

(3) Choose a small constant $\alpha$ to control the efficiency loss at $\rho_{1}$ due to the introduction of parameter $k$. Similar to Shu [23], $\alpha=0.05$ is also chosen for discussion in this paper.

(4) Set the smoothing parameter $\lambda$ of the AEWMA TBE chart to be the same as the one obtained in Step (2), then find the optimal parameters $k$ and $U C L^{+}$or $L C L^{-}$of the AEWMA TBE charts that have the minimum $A R L_{1}$ value at $\rho_{2}$ under the constraint that the percentage increase in $A R L_{1}$ at $\rho_{1}$ is smaller than $\alpha$.

It can be seen that the optimal parameter $\lambda$ of the EWMA chart is used as the approximately optimal one for the AEWMA chart. This is due to the attractive property of the parameter $k$ which can be defined in order to increase the AEWMA chart's performance at large shifts, with a large percentage, while causing only a small loss in the chart's performance at small shifts (see Shu [23]). Moreover, it should be pointed out that the above "nearly" optimal design procedure is not intended for the detection of any particular level of shifts. We will show lately that these design approach can provide a reasonably good detection performance over the specified range of shifts $\left[\rho_{1}, \rho_{2}\right]$.

For different values of the desired $A R L_{0}$ and the range of shifts $\left[\rho_{1}, \rho_{2}\right]$, Tables 1 and 2 present the optimal parameters of the upper- and lower-sided AEWMA TBE charts, respectively. These parameters are optimized using the foregoing procedures. For example, in Table 1 , when $A R L_{0}=200$ and $r=1$, if a practitioner expects a good overall performance of the upper-sided AEWMA chart for shifts within the range $[1.2,10]$, then the "nearly" optimal parameters are $\left(\lambda, k, U C L^{+}\right)=(0.02,4.900,1.2063)$. Here, the parameter $\lambda=0.02$ is first obtained by minimizing the $A R L_{1}$ of the upper-sided EWMA TBE chart for a small shift $\rho_{1}=1.2$, with the constraint on the desired $A R L_{0}$. This gives the minimum $A R L_{1}=54.4766$ of the upper-sided EWMA TBE chart for $\rho_{1}=1.2$. Then if we choose a constant $\alpha=0.05$ and set the parameter $\lambda=0.02$ in the upper-sided AEWMA TBE chart, the optimal parameters $\left(k, U C L^{+}\right)=(4.900,1.2063)$ are obtained by minimizing the $A R L_{1}$ of the upper-sided AEWMA TBE chart for a large shift $\rho_{2}=10$, with the constraint that the desired $A R L_{0}$ is satisfied and the $A R L_{1}$ of the AEWMA chart for $\rho_{1}$ is smaller than $(1+\alpha) \times 54.4766=57.2004$. For other cases in Tables 1 and 2 , the optimal parameters $\left(\lambda, k, U C L^{+}\right)$can be obtained similarly. 
From Tables 1 and 2, several conclusions concerning the optimal parameters of the one-sided AEWMA TBE charts can be drawn as follows,

- For fixed range of shifts $\left[\rho_{1}, \rho_{2}\right]$ and $A R L_{0}$, the optimal parameters $\left(\lambda, k, U C L^{+}\right)$ or $\left(\lambda, k, U C L^{-}\right)$tends to increase when $r$ increases, with the exception of the case $\rho_{1}=2.5$ and $\rho_{2}=5$, where no obvious trend of $k$ can be noted. For example, in Table 1, when $\rho_{1}=1.2, \rho_{2}=8, A R L_{0}=200$ and $r$ increases from 1 up to 4, the optimal parameter $\lambda$ increases from 0.02 up to $0.05, k$ increases from 4.900 up to 7.775 and $U C L^{+}$increases from 1.2063 up to 4.7800 , respectively.

- For fixed values of $r, A R L_{0}$ and $\rho_{2}$, the optimal parameters $\lambda$ tends to increase and $k$ tends to decrease when $\rho_{1}$ increases. For example, in Table 1 , when $A R L_{0}=200, r=1$ and $\rho_{2}=5$, the optimal parameter $\lambda$ increases from $0.02 \mathrm{up}$ to 0.17 and $k$ decreases from 4.900 down to 4.250 as the parameter $\rho_{1}$ increases from 1.2 up to 2.5. This may be due to the fact that a large value of $\lambda$ or a small value of $k$ is more sensitive to large changes in $\rho_{1}$. This is also consistent with the results in Capizzi and Masarotto [22] and Shu [23].

- For fixed values of $r, \rho_{1}$ and $\rho_{2}$, the optimal parameter $\lambda$ tends to decrease and $k$ tends to increase when the desired $A R L_{0}$ increases. For example, in Table 1, when $r=1, \rho_{1}=1.2$ and $\rho_{2}=5$, the optimal parameter $\lambda$ decreases from 0.02 down to 0.01 while $k$ increases from 4.900 up to 6.125 as $A R L_{0}$ increases from 200 up to 500 .

\subsection{Comparisons with one-sided EWMA and Shewhart TBE charts}

To study the performance of one-sided AEWMA TBE charts, for some combinations of the design parameters $\left(\lambda, k, U C L^{+}\right)$and $\left(\lambda, k, L C L^{-}\right)$presented in Tables 1 and 2, $A R L_{1}$ values of one-sided AEWMA TBE charts are shown in Tables 3 to 10 when $A R L_{0}=200$ and $r \in\{1,2,3,4\}$. For comparisons, some $A R L_{1}$ values of one-sided EWMA and Shewhart TBE charts are also presented in these tables. In our study, the optimal parameters of the upper-sided AEWMA chart are designed for the range of shifts $\left[\rho_{1}, \rho_{2}\right]$, where a small increasing shift $\rho_{1} \in\{1.2,1.5,2,2.5\}$ (see the bolded entry in each table) and a large increasing shift $\rho_{2}=8$. For the lower-sided AEWMA chart, we consider the optimal parameters designed for the range of shifts $\left[\rho_{1}, \rho_{2}\right]$, where a small decreasing shift $\rho_{1} \in\{0.9,0.8,0.7,0.6\}$ (see the bolded entry in each table) and a large decreasing shift $\rho_{2}=0.1$. For example, in Table 3 , for $r=1$, the optimal parameters $\left(\lambda, k, U C L^{+}\right)=(0.02,4.900,1.2063)$ of the upper-sided AEWMA chart are obtained following the sequential searching procedure presented in Section 3.2 when $\left[\rho_{1}, \rho_{2}\right]=[1.2,8]$. Due to the space limitation, $A R L_{1}$ values of the upper-sided AEWMA TBE charts for shifts larger than 5 are not listed in Tables 3 to 6 .

\section{(Please Insert Tables Tables 3 to 10 Here)}

From these tables, it can be generally concluded that, for small increasing or small decreasing shifts in the process, the proposed AEWMA TBE schemes perform much better than the Shewhart TBE charts and perform similarly to the EWMA TBE charts. For large increasing or decreasing shifts in the process, the AEWMA schemes perform better than the EWMA TBE charts and perform similarly to the Shewhart TBE charts. Some detailed conclusions are listed as follows, 
- For shifts increasing from $\rho_{1}$ up to $\rho_{2}$, the upper-sided AEWMA TBE chart either performs better than the upper-sided EWMA chart or the upper-sided Shewhart chart. For instance, in Table 3, when $r=1, \rho_{1}=1.2$ and the designed parameter $\left(\lambda, k, U C L^{+}\right)$are $(0.02,4.900,1.2063)$, it can be seen that $A R L_{1}$ values of the upper-sided AEWMA chart are smaller than the upper-sided EWMA chart when the shifts are in the range [1.8,5]. For shifts in the range [1.2,3.3], the $A R L_{1}$ values of the upper-sided AEWMA chart are all smaller than the ones of the upper-sided Shewhart chart. Similar conclusions also hold for other cases presented in Tables 3 to 6 .

- As $r$ increases, the range of shifts for which the upper-sided EWMA scheme performs better than the upper-sided AEWMA TBE chart becomes narrower, which also means that the AEWMA scheme performs better than the EWMA scheme in a wider range of shifts. For example, the range of shifts for which the AEWMA chart is better than the EWMA chart changes from $[1.8,5]$ to $[1.5,5]$ as $r$ increases from 1 up to 4 (see columns 3 to 5 and 12 to 14 in Table 3). Similar conclusions also hold for other cases in Tables 4 to 6 .

As $r$ increases, the range of shifts for which the upper-sided Shewhart scheme performs a little better than the upper-sided AEWMA TBE chart becomes wider. While the upper-sided Shewhart TBE chart performs much worse than the upper-sided AEWMA TBE chart for small increasing shifts. For example, when $r$ increases from 1 to 3 , the range of shifts for which the upper-sided Shewhart chart performs a little better than the upper-sided AEWMA chart changes from $[3.4,5]$ to $[2.5,5]$ (see columns 3 to 5 and 9 to 11 in Table 3), while for a small increasing shift, like $\rho=1.4$, the $A R L_{1}=44.01$ of the upper-sided Shewhart chart is much larger than $A R L_{1}=29.24$ of the upper-sided AEWMA chart when $r=1$. Similar conclusions also hold for other cases in Tables 4 to 6 .

- For shifts decreasing from $\rho_{1}$ down to $\rho_{2}$, the lower-sided AEWMA TBE chart either performs better than the lower-sided EWMA TBE chart or the lowersided Shewhart TBE chart. For instance, in Table 7, when $r=2, \rho_{1}=0.9$ and the optimal parameters $\left(\lambda, k, L C L^{-}\right)$are $(0.01,2.425,1.8773)$, it can be seen that $A R L_{1}$ values of the lower-sided AEWMA chart are smaller than that of the lower-sided EWMA chart when the decreasing shifts are smaller than 0.8. For decreasing shifts from 0.9 down to 0.175 , the $A R L_{1}$ values of the lower-sided AEWMA chart are all smaller than the ones of the lower-sided Shewhart chart. Similar conclusions also hold for other cases presented in Tables 7 to 10.

- As $r$ increases, the range of shifts for which the lower-sided Shewhart scheme performs a little better than the lower-sided AEWMA TBE chart becomes wider. While the lower-sided Shewhart TBE chart performs much worse than the lowersided AEWMA TBE chart for small decreasing shifts. For example, when $r$ increases from 2 to 4 in Table 7, the range of shifts for which the lower-sided Shewhart chart performs a little better than the lower-sided AEWMA chart changes from $[0.1,0.15]$ to $[0.1,0.325]$ (see columns 6 to 8 and 12 to 14 in Table 7), while for a small decreasing shift, like $\rho=0.85$, the $A R L_{1}=146.25$ of the lower-sided Shewhart chart is much larger than $A R L_{1}=53.21$ of the lowersided AEWMA chart when $r=2$. Similar conclusions also hold for other cases in Tables 8 to 10.

- For a fixed increasing or decreasing shift $\rho$, the $A R L_{1}$ values of the one-sided AEWMA, the one-sided EWMA and the Shewhart TBE charts decrease as the value of $r$ increases. For example, in Table 3, as $r$ increases from 1 up to 4, the $A R L_{1}$ values of the upper-sided AEWMA TBE chart decreases from 57.19 
down to 28.24 for a shift $\rho=1.2$. While, in practice, one has to be careful when using a large value of $r$ because this means that more observations should be collected to check the status of the process. This should depend on how long one can reasonably wait in a given context before a decision has to be made (see Xie et al. [11]).

\subsection{Comparisons with one-sided run rules Shewhart TBE charts}

From the research works in Section 3.3, it can be seen that the one-sided AEWMA TBE charts perform much better than the one-sided Shewhart TBE charts for small shifts in the process. While it it known that the Shewhart TBE chart's performance for the detection of small to moderate shifts can be improved by applying different run rules schemes, see Cheng and Chen [42], Santiago and Smith [43], Rakitzis [44] and Kumar et al. [45] and so on. Several run rule schemes were suggested in these research works, which were only focused on two-sided Shewhart TBE charts. As we are concerned with one-sided type charts, we suggest to study the performance of the one-sided run rules Shewhart TBE charts and make some comparisons with one-sided AEWMA and Shewhart TBE charts. Concerning one-sided run rules Shewhart charts, Amdouni et al. [46] proposed one-sided 2-of-3 and 3-of-4 Shewhart charts for monitoring coefficient of variation in a short production run. These run rules can be concluded as: an out-of-control signal is given if the last $n$ of $m$ monitoring statistics fall in the interval $(a, b)$, where $n \leq m$ and $a<b$. Motivated by this, the performance of one-sided Shewhart TBE charts with 2-of-3, 3-of-4 and 4-of-5 run rules are also presented in this section as a comparison with the proposed one-sided AEWMA TBE charts. A Markov chain approach is used to obtain the run length properties of the run rules Shewhart TBE charts. More details can be found in Amdouni et al. [46], Castagliola et al. [47] and Tran [48].

The $A R L \mathrm{~s}$ of the aforementioned run rules one-sided Shewhart TBE schemes are given in Tables 11 and 12, along with the proposed one-sided AEWMA TBE charts and the traditional one-sided Shewhart TBE charts. The 2-of-3, 3-of-4 and 4-of-5 run rules TBE charts are denoted as $R R_{2,3}, R R_{3,4}$ and $R R_{4,5}$ in these tables. The optimal parameters $\left(\lambda, k, U C L^{+}\right)$and $\left(\lambda, k, L C L^{-}\right)$of the one-sided AEWMA TBE charts are from Tables 3 and 7 . Control limits of all other charts are selected to ensure $A R L_{0}=200$. It follows from Table 11 that all the upper-sided run rules Shewhart TBE charts perform worse than the upper-sided AEWMA TBE chart. For some smaller increasing shifts $(\rho<1.5)$, run rules Shewhart TBE charts perform better than the chart without run rules. For shifts larger than 1.5, the upper-sided Shewhart TBE chart generally performs better than the chart with run rules. For example, when $r=2$ and $\rho=1.5$, the $A R L_{1}=21.89$ of the 2 -of- 3 run rules chart is smaller than $A R L_{1}=23.79$ of the Shewhart chart. While, if $\rho=2.5$, the $A R L_{1}=5.53$ of the 2 -of-3 run rules chart is larger than $A R L_{1}=4.92$ of the Shewhart chart.

Considering lower-sided TBE charts in Table 12, for shifts decreasing from 1 down to $0.6(0.6 \leq \rho<1)$, the lower-sided AEWMA TBE chart performs better than all the lower-sided run rules Shewhart TBE charts. While, for moderate to large decreasing shifts, especially when $r$ is large, lower-sided Shewhart TBE chart with or without run rules perform better than the lower-sided AEWMA TBE chart. For example when $r=4$, if $0.15 \leq \rho \leq 0.575$, the 4 -of- 5 run rules 
Shewhart TBE chart performs better than the lower-sided AEWMA TBE chart. If $0.125 \leq \rho \leq 0.475$, both the 2-of-3 and 3-of-4 run rules Shewhart TBE charts perform better than the lower-sided AEWMA TBE chart. Moreover, it is pointed out that, for most decreasing shifts except for some large decreasing shifts, lower-sided Shewhart TBE charts with run rules perform uniformly better than the charts without run rules.

(Please Insert Tables 11 to 12 Here)

\subsection{Comparisons with one-sided DEWMA TBE charts}

In this section, one-sided Double EWMA (DEWMA) TBE charts presented in Alevizakos and Koukouvinos [49] are briefly introduced as a comparison with the proposed one-sided AEWMA TBE charts. The upper-sided DEWMA and lowersided DEWMA TBE charts are constructed by plotting only one charting statistic $Z_{t}=\lambda W_{t}+(1-\lambda) Z_{t-1}\left(Z_{0}=r \theta_{0}\right)$, where $W_{t}=\lambda X_{t}+(1-\lambda) W_{t-1}\left(W_{0}=r \theta_{0}\right)$. To detect an increasing or a decreasing shift in the process, the statistic $Z_{t}$ is compared with an upper control limit $\left(U C L^{+}\right)$or a lower control limit $\left(L C L^{-}\right)$, respectively. An out-of-control signal is triggered when the charting statistic $Z_{t}$ falls above $U C L^{+}$ (for the upper-sided chart) or below $L C L^{-}$(for the lower-sided chart). For more details on the properties of the one-sided DEWMA TBE charts, reader can refer to Alevizakos and Koukouvinos [49].

The $A R L$ s of the one-sided DEWMA TBE charts and the proposed one-sided AEWMA TBE charts are presented in Tables 13 and 14. The optimal parameters $\left(\lambda, k, U C L^{+}\right)$and $\left(\lambda, k, L C L^{-}\right)$of the one-sided AEWMA TBE charts are from Tables 3 and 7 . Moreover, the smoothing parameter $\lambda$ in the one-sided DEWMA TBE charts are set to be the same as the ones of the one-sided AEWMA TBE charts and the control limits of the DEWMA charts are selected to maintain $A R L_{0}=200$.

It can be seen from Table 13 that the upper-sided AEWMA TBE chart performs better than the upper-sided DEWMA TBE chart for the detection of large shifts in the process. While for the detection of small shifts in the process, the upper-sided DEWMA TBE chart is better than the upper-sided AEWMA TBE chart. For example, when $r=2$, if $\rho \geq 1.4$, the $A R L_{1}$ values of the upper-sided AEWMA TBE chart are smaller than the ones of the upper-sided DEWMA TBE chart. While for the detection of small shifts $(\rho \leq 1.3)$, the $A R L_{1}$ values of the upper-sided DEWMA TBE chart are smaller than the ones of the upper-sided AEWMA TBE chart. In addition, for other optimal parameters $\left(\lambda, k, U C L^{+}\right)$of the upper-sided AEWMA TBE charts from Tables 4 to 6 , similar conclusions can also be drawn from the $A R L_{1}$ comparison of two upper-sided TBE charts. For simplicity, these results are not presented here. Moreover, these conclusions drawn for the upper-sided TBE charts also apply for the lower-sided AEWMA TBE chart and the lowersided DEWMA TBE chart. For example, in Table 14, when $r=2$, if $\rho \leq 0.350$, the $A R L_{1}$ values of the lower-sided AEWMA TBE chart are smaller than the ones of the lower-sided DEWMA TBE chart. While for other values of $\rho$, the lowersided DEWMA TBE chart performs better than the lower-sided AEWMA TBE chart.

(Please Insert Tables 13 to 14 Here) 


\section{A real data example}

In this section, a real data example from Hellenic Air Force (HAF) presented in Alevizakos and Koukouvinos [49] is used to illustrate the implementation of the proposed charts. As a critical quality characteristic, the time $X$ between consecutive accidents of the F-16 supplied by Greece from 1988 to 2017 are recorded in Table 15. It can be noted that the first accident happened at 1456 days after the first accept of F-16. It is assumed that $\theta_{0}=1500$, which means that the process is in-control if an accident occurs about every four years on average. By using the chi-square goodness-of-fit test, it has been tested that the TBE data in Table 15 follows a Gamma(1,615) distribution, see Alevizakos and Koukouvinos [49]. Then the specified decreasing shift is $\rho=615 / 1500=0.41$ in the out-of-control process in this case.

\section{(Please Insert Table 15 Here)}

In order to give good protection against the shift $\rho=0.41$, the lower-sided AEWMA TBE chart is designed for the shift range [0.6,0.1], leading to the optimal parameters $\left(\lambda, k, L C L^{-}\right)=(0.07,0.9,0.6544)$ when $A R L_{0}=200$ and $r=1$ (see Table $2)$. As a comparison, the designed parameters $\left(\lambda, L C L^{-}\right)$of the lower-sided EWMA TBE chart and lower-sided DEWMA TBE chart are $(0.07,0.6414)$ and $(0.07,0.8326)$, respectively. In addition, if Shewhart TBE charts are used for the process monitoring, the lower-sided Shewhart TBE chart with 4-of-5 run rules is suggested as it performs better than the Shewhart TBE chart with 2-of-3 or 3-of-4 run rules for the detection of a shift size $\rho=0.41$. It can be noted from Table 12 that the designed control limits $L C L^{-}$of the lower-sided Shewhart TBE chart without and with 4-of-5 run rules are 0.005 and 0.2425 , respectively.

Since all the conducted discussions in this paper assume $\theta_{0}=1$, the real dataset in Table 15 has to be scaled by dividing them by 1500 . These scaled data are presented in Table 16 along with the corresponding charting statistics of the lower-sided AEWMA, EWMA and DEWMA TBE charts. From Table 16, it can be noted that, at time $t=12$, the statistic $Z_{12}^{-}=0.6534$ (see the bolded entry) of the lower-sided AEWMA chart is smaller than $L C L^{-}=0.6544$, which implies that a decreasing shift in the process happened. For the lower-sided EWMA and DEWMA TBE charts, the $13 t h$ and $15 t h$ charting statistics $Z_{13}^{-}=0.6286$ and $Z_{15}=0.8228$ (see the bolded entries) are smaller than the corresponding control limits $L C L^{-}=0.6414$ and $L C L^{-}=0.8326$, respectively.

\section{(Please Insert Table 16 Here)}

Figure 2 displays the corresponding lower-sided AEWMA, EWMA and DEWMA TBE charts, along with the Shewhart TBE chart with and without run rules, for the real dataset. As it can be seen from graphs (a), (b) and (c) in Figure 2, these three charts detect the shift after the process running for some time and the AEWMA TBE chart detects the decreasing shift in the process a little quicker than the EWMA and DEWMA TBE charts. While we can also observe from the graph (d) that no point is below the $L C L^{-}$of the Shewhart TBE chart and from the graph (e) that, points 2,4,7,15 and 16 are below the $L C L^{-}$of the 4 -of-5 run rules Shewhart TBE chart. Considering the schemes of the Shewhart type charts with- or without run rules, 
there is no shift detected by Shewhart TBE charts.

It is pointed out that the advantage of the AEWMA TBE chart is for the detection of a wider range of shifts. If the actual shift size is not $\rho=0.41$, say for example $\rho=0.3$, the $A R L_{1}$ values of AEWMA, EWMA, DEWMA, Shewhart and 4-of-5 run rules Shewhart TBE charts are obtained as 9.57, 10.44, 12.95, 60.35 and 11.97, respectively. This also shows the advantage of the AEWMA TBE chart.

\section{(Please Insert Figure 2 Here)}

\section{Conclusions and recommendations}

In this article, one-sided AEWMA charts are proposed for monitoring TBE data based on the Gamma distribution. The proposed one-sided AEWMA TBE charts can be seen as a smooth combination of a Shewhart TBE chart and an EWMA TBE chart. A Markov chain approach is used to obtain the run length properties of the chart and the "nearly" optimal parameters of the one-sided AEWMA TBE charts are presented. We also compared the performance of the proposed schemes with the one-sided EWMA and one-sided Shewhart TBE charts. The numerical results showed that the one-sided AEWMA TBE charts either perform better than the EWMA TBE chart for moderate to large shifts or perform better than the Shewhart TBE chart for small shifts. Compared with Shewhart TBE charts, the losses of one-sided AEWMA charts for the detection of large shifts is negligible compared with the improvement for the detection of small shifts. Moreover, through the comparison of the one-sided AEWMA TBE chart and the one-sided DEWMA TBE chart, it can be concluded that the one-sided AEWMA TBE chart performs better than the one-sided DEWMA TBE chart for the detection of large shifts in the process. While for the detection of very small shifts in the process, the one-sided DEWMA TBE chart performs better than the one-sided AEWMA TBE chart. Finally, we applied the AEWMA TBE chart to a real example.

It should be pointed out that many other issues can also be studied in future research works. For instance, other one-sided modified AEWMA control charts can be used to monitor the TBE data and the properties of the proposed scheme need to be further investigated when the process parameters are estimated from in-control samples. In addition, when two one-sided AEWMA TBE charts are simultaneously used or a two-sided AEWMA TBE chart is used, the $A R L$-biased problem will be investigated in the future.

\section{References}

[1] Douglas C. Montgomery. Intorduction to Ststistical Quality Control. Wiley, the seventh edition edition, 2009.

[2] F.F. Gan. Designs of one- and two-sided exponential EWMA charts. Journal of Quality Technology, 30(1):55-69, 1998.

[3] C.W. Zhang, M. Xie, J.Y. Liu, and T.N. Goh. A control chart for the Gamma distribution 
as a model of time between events. International Journal Production Research, 45(23): 5649-5666, 2007.

[4] M Xie, T N Goh, and V Kuralmani. Statistical Models and Control Charts for HighQuality Processes. Springer, Boston, MA, 2002.

[5] Y.J. Xie, K.L. Tsui, M. Xie, and T.N. Goh. Monitoring time-between-events for health management. In 2010 Prognostics and System Health Management Conference. IEEE, jan 2010. .

[6] Eduardo Santiago and Joel Smith. Control charts based on the exponential distribution: Adapting runs rules for the $t$ chart. Quality Engineering, 25(2):85-96, 2013.

[7] Dorra Rahali, Philippe Castagliola, Hassen Taleb, and Michael B. C. Khoo. Evaluation of Shewhart time-between-events-and-amplitude control charts for several distributions. Quality Engineering, 31(2):240-254, 2019.

[8] A.C. Rakitzis. Monitoring exponential data using two-sided control charts with runs rules. Journal of Statistical Computation and Simulation, 86(1):149-159, 2016.

[9] Nirpeksh Kumar and Subhabrata Chakraborti. Phase II Shewhart-type control charts for monitoring times between events and effects of parameter estimation. Quality and Reliability Engineering International, 32:315-328, 2016.

[10] Nirpeksh Kumar. Conditional analysis of Phase II exponential chart for monitoring times to an event. Quality Technology \& Quantitative Management, 2019. .

[11] M. Xie, T.N. Goh, and P. Ranjan. Some effective control chart procedures for reliability monitoring. Reliability Engineering \& System Safety, 77(2):143 - 150, 2002.

[12] S. Ali. Time-betwee-events control charts for an exponentiated class of distributions of the renewal process. Quality and Reliability Engineering International, 33(8):2625-2651, 2017.

[13] C.W. Zhang, M. Xie, and T.N. Goh. Design of exponential control charts using a sequential sampling scheme. IISE Transactions, 38:1105-1116, 2006.

[14] J. Yang, H. Yu, Y. Cheng, and M. Xie. Design of gamma charts based on average time to signal. Quality and Reliability Engineering International, 32:1041-1058, 2016.

[15] S. Ali and A. Pievatolo. High quality process monitoring using a class of inter-arrival time distributions of the renewal process. Computers \& Industrial Engineering, 94:45-62, 2016.

[16] S. Ali and I. Shah. Monitoring regularly maintained systems based on the renewal process with generalized exponential distribution of time between events. Journal of Testing and Evaluation, 48(5):3673-3694, 2020.

[17] S. Ali, I. Shah, L.C. Wang, and Z. Yue. A comparison of shewhart-type time-betweenevents control charts based on the renewal process. IEEE Access, 8:113683-113701, 2020.

[18] N. Khan, M. Aslam, and C.H. Jun. A EWMA control chart for exponential distributed quality based on moving average statistics. Quality and Reliability Engineering International, 32:1179-1190, 2016.

[19] J.Y. Liu, M. Xie, and T.N. Goh. CUSUM chart with transformed exponential data. Communications in Statistics-Theory and Methods, 35:1829-1843, 2006.

[20] Liang Qu, Michael B.C. Khoo, Philippe Castagliola, and Zhen He. Exponential cumulative sums chart for detecting shifts in time-between-events. International Journal of Production Research, 56(10):3683-3698, 2018.

[21] Sajid Ali, Antonio Pievatolo, and Rainer Göb. An overview of control charts for highquality processes. Quality and Reliability Engineering International, 32(7):2171-2189, 2016.

[22] G. Capizzi and G. Masarotto. An adaptive exponentially weighted moving average control chart. Technometrics, 45(3):199-207, 2003.

[23] Lianjie Shu. An adaptive exponentially weighted moving average control chart for monitoring process variances. Journal of Statistical Computation and Simulation, 78(4):367$384,2008$.

[24] Willy Ugaz, Ismael Sánchez, and Andrés M. Alonso. Adaptive EWMA control charts with time-varying smoothing parameter. The International Journal of Advanced Manu- 
facturing Technology, 93(9-12):3847-3858, 2017.

[25] Anan Tang, Philippe Castagliola, JinSheng Sun, and XueLong Hu. The effect of measurement errors on the adaptive EWMA chart. Quality and Reliability Engineering International, 34(4):609-630, 2018.

[26] Anan Tang, Philippe Castagliola, Xuelong Hu, and Jinsheng Sun. The performance of the adaptive EWMA median chart in the presence of measurement error. Quality and Reliability Engineering International, 35(1):423-438, 2019.

[27] Nesma A. Saleh, Mahmoud A. Mahmoud, and Abdel-Salam G. Abdel-Salam. The performance of the adaptive exponentially weighted moving average control chart with estimated parameters. Quality and Reliability Engineering International, 29(4):595-606, 2013.

[28] Aya A. Aly, Nesma A. Saleh, Mahmoud A. Mahmoud, and William H. Woodall. A reevaluation of the adaptive exponentially weighted moving average control chart when parameters are estimated. Quality and Reliability Engineering International, 31(8):1611$1622,2015$.

[29] Anan Tang, Jinsheng Sun, Xuelong Hu, and Philippe Castagliola. A new nonparametric adaptive EWMA control chart with exact run length properties. Computers \& Industrial Engineering, 130:404 - 419, 2019.

[30] Aya A. Aly, Nesma A. Saleh, and Mahmoud A. Mahmoud. An adaptive EWMA control chart for monitoring zero-inflated poisson processes. Communications in Statistics Simulation and Computation, 0(0):1-14, 2019.

[31] Anan Tang, Philippe Castagliola, Jinsheng Sun, and Xuelong Hu. Optimal design of the adaptive EWMA chart for the mean based on median run length and expected median run length. Quality Technology \& Quantitative Management, 16(4):439-458, 2019.

[32] Anan Tang, Philippe Castagliola, Xuelong Hu, and Jinsheng Sun. The adaptive EWMA median chart for known and estimated parameters. Journal of Statistical Computation and Simulation, 89(5):844-863, 2019.

[33] Cesar A. Acosta-Mejia and Joseph J. Pignatiello Jr. ARL-design of $S$ charts with $k$-of- $k$ runs rules. Communications in Statistics-Simulation and Computation, 38(8):1625-1639, 2009.

[34] Manuel Cabral Morais, Sven Knoth, and Christian H. Weiß. An ARL-unbiased thinningbased EWMA chart to monitor counts. SEQUENTIAL ANALYSIS, 37(4):487-510, 2018.

[35] Philippe Castagliola, Giovanni Celano, and Stelios Psarakis. Monitoring the coefficient of variation using EWMA charts. Journal of Quality Technology, 43(3):249-265, 2011.

[36] Jiujun Zhang, Zhonghua Li, Bin Chen, and Zhaojun Wang. A new exponentially weighted moving average control chart for monitoring the coefficient of variation. Computers \& Industrial Engineering, 78:205-212, 2014.

[37] Kim Phuc Tran and Sven Knoth. Steady-state ARL analysis of ARL-unbiased EWMARZ control chart monitoring the ratio of two normal variables. Quality and Reliability Engineering International, 34(3):377-390, 2018.

[38] J. Lucas and M. Saccucci. Exponentially weighted moving average control schemes: properties and enhancements. Journal of Quality Technology, 32(1):1-12, 1990.

[39] M. Neuts. Matrix-Geometric Solutions in Stochastic Models: an Algorithmic Approach. Dover Publications Inc, 1981.

[40] G. Latouche and V. Ramaswami. Introduction to Matrix Analytic Methods in Stochastic Modelling. ASA-SIAM, 1999.

[41] Yan Su, Lianjie Shu, and Kwok-Leung Tsui. Adaptive EWMA procedures for monitoring processes subject to linear drifts. Computational Statistics and Data Analysis, 55:28192829, 2011.

[42] Chuen-Sheng Cheng and Pei-Wen Chen. An ARL-unbiased design of time-between-events control charts with runs rules. Journal of Statistical Computation and Simulation, 81(7): 857-871, 2011. .

[43] Eduardo Santiago and Joel Smith. Control charts based on the exponential distribution: Adapting runs rules for the $t$ chart. Quality Engineering, 25(2):85-96, 2013. .

[44] Athanasios C. Rakitzis. Monitoring exponential data using two-sided control charts with 
runs rules. Journal of Statistical Computation and Simulation, 86(1):149-159, 2016. .

[45] N. Kumar, S. Chakraborti, and A. C. Rakitzis. Improved Shewhart-type charts for monitoring times between events. Journal of Quality Technology, 49(3):278-296, 2017. .

[46] Asma Amdouni, Philippe Castagliola, Hassen Taleb, and Giovanni Celano. One-sided run rules control charts for monitoring the coefficient of variation in short production runs. European Journal of Industrial Engineering, 10(5):639-662, 2016.

[47] Philippe Castagliola, Ali Achouri, Hassen Taleb, Giovanni Celano, and Stelios Psarakis. Monitoring the coefficient of variation using control charts with run rules. Quality Technology \& Quantitative Management, 10(1):75-94, 2013.

[48] Kim Phuc Tran. Designing of run rules $t$ control charts for monitoring changes in the process mean. Chemometrics and Intelligent Laboratory Systems, 174:85-93, 2018.

[49] Vasileios Alevizakos and Christos Koukouvinos. A double exponentially weighted moving average chart for time between events. Communications in Statistics-Simulation and Computation, 2019. 
Table 1. Optimal parameters of the upper-sided AEWMA TBE chart for shift range $\left[\rho_{1}, \rho_{2}\right]$ when $A R L_{0} \in$ $\{200,370,500\}$ and $r \in\{1,2,3,4\}$

\begin{tabular}{|c|c|c|c|c|c|c|c|c|c|c|c|c|c|}
\hline \multirow[b]{3}{*}{$\rho_{1}$} & \multirow[b]{3}{*}{$\rho_{2}$} & \multicolumn{12}{|c|}{$A R L_{0}=200$} \\
\hline & & \multicolumn{3}{|c|}{$r=1$} & \multicolumn{3}{|c|}{$r=2$} & \multicolumn{3}{|c|}{$r=3$} & \multicolumn{3}{|c|}{$r=4$} \\
\hline & & $\lambda$ & $k$ & $U C L^{+}$ & $\lambda$ & $k$ & $U C L^{+}$ & $\lambda$ & $k$ & $U C L^{+}$ & $\lambda$ & $k$ & $U C L^{+}$ \\
\hline 1.2 & $\{5,8,10,13\}$ & 0.02 & 4.900 & 1.2063 & 0.03 & 6.175 & 2.3883 & 0.04 & 7.075 & 3.5800 & 0.05 & 7.775 & 4.7800 \\
\hline 1.5 & $\{5,8,10,13\}$ & 0.05 & 4.650 & 1.4230 & 0.09 & 5.725 & 2.8828 & 0.11 & 6.400 & 4.2330 & 0.14 & 6.950 & 5.6628 \\
\hline 2 & $\{5,8,10,13\}$ & 0.11 & 4.300 & 1.7885 & 0.18 & 5.100 & 3.5394 & 0.25 & 5.550 & 5.3477 & 0.30 & 5.825 & 7.0813 \\
\hline \multirow[t]{2}{*}{2.5} & $\{8,10,13\}$ & 0.17 & 4.025 & 2.1370 & 0.28 & 4.600 & 4.2430 & 0.37 & 4.675 & 6.3942 & 0.45 & 4.225 & 8.6824 \\
\hline & 5 & 0.17 & 4.250 & 2.0761 & 0.28 & 4.725 & 4.1883 & 0.37 & 4.675 & 6.3942 & 0.45 & 4.550 & 8.5146 \\
\hline$\rho_{1}$ & $\rho_{2}$ & \multicolumn{12}{|c|}{$A R L_{0}=370$} \\
\hline 1.2 & $\{5,8,10,13\}$ & 0.01 & 5.725 & 1.1427 & 0.02 & 7.075 & 2.3332 & 0.03 & 8.000 & 3.5398 & 0.04 & 8.775 & 4.7541 \\
\hline 1.5 & $\{5,8,10,13\}$ & 0.04 & 5.425 & 1.4074 & 0.07 & 6.550 & 2.8278 & 0.10 & 7.325 & 4.2697 & 0.12 & 7.925 & 5.6408 \\
\hline 2 & $\{5,8,10,13\}$ & 0.09 & 5.050 & 1.7553 & 0.16 & 5.950 & 3.5384 & 0.21 & 6.475 & 5.2488 & 0.26 & 6.850 & 6.9887 \\
\hline \multirow[t]{2}{*}{2.5} & $\{8,10,13\}$ & 0.14 & 4.775 & 2.0778 & 0.24 & 5.475 & 4.1598 & 0.32 & 5.750 & 6.2372 & 0.39 & 5.700 & 8.3706 \\
\hline & 5 & 0.14 & 4.950 & 2.0425 & 0.24 & 5.525 & 4.1413 & 0.32 & 5.750 & 6.2372 & 0.39 & 5.700 & 8.3706 \\
\hline$\rho_{1}$ & $\rho_{2}$ & \multicolumn{12}{|c|}{$A R L_{0}=500$} \\
\hline 1.2 & $\{5,8,10,13\}$ & 0.01 & 6.125 & 1.1541 & 0.02 & 7.500 & 2.3550 & 0.03 & 8.450 & 3.5711 & 0.03 & 9.200 & 4.6573 \\
\hline 1.5 & $\{5,8,10,13\}$ & 0.04 & 5.825 & 1.4289 & 0.07 & 6.975 & 2.8656 & 0.09 & 7.750 & 4.2369 & 0.11 & 8.375 & 5.6148 \\
\hline 2 & $\{5,8,10,13\}$ & 0.09 & 5.400 & 1.7908 & 0.15 & 6.350 & 3.5301 & 0.20 & 6.925 & 5.2472 & 0.24 & 7.325 & 6.9249 \\
\hline \multirow[t]{2}{*}{2.5} & $\{8,10,13\}$ & 0.13 & 5.125 & 2.0663 & 0.22 & 5.875 & 4.1074 & 0.30 & 6.225 & 6.1837 & 0.37 & 6.275 & 8.3035 \\
\hline & 5 & 0.13 & 5.300 & 2.0353 & 0.22 & 5.925 & 4.0904 & 0.30 & 6.225 & 6.1837 & 0.37 & 6.275 & 8.3035 \\
\hline
\end{tabular}

Table 2. Optimal parameters of the lower-sided AEWMA TBE chart for shift range $\left[\rho_{1}, \rho_{2}\right]$ when $A R L_{0} \in$ $\{200,370,500\}$ and $r \in\{1,2,3,4\}$

\begin{tabular}{|c|c|c|c|c|c|c|c|c|c|c|c|c|c|}
\hline \multirow[b]{3}{*}{$\rho_{1}$} & \multirow[b]{3}{*}{$\rho_{2}$} & \multicolumn{12}{|c|}{$A R L_{0}=200$} \\
\hline & & \multicolumn{3}{|c|}{$r=1$} & \multicolumn{3}{|c|}{$r=2$} & \multicolumn{3}{|c|}{$r=3$} & \multicolumn{3}{|c|}{$r=4$} \\
\hline & & $\lambda$ & $k$ & $L C L^{-}$ & $\lambda$ & $k$ & $L C L^{-}$ & $\lambda$ & $k$ & $L C L^{-}$ & $\lambda$ & $k$ & $L C L^{-}$ \\
\hline 0.9 & $\{0.3,0.2,0.1\}$ & 0.01 & 1.775 & 0.9139 & 0.01 & 2.425 & 1.8773 & 0.02 & 2.925 & 2.7291 & 0.02 & 3.525 & 3.6793 \\
\hline 0.8 & $\{0.3,0.2,0.1\}$ & 0.02 & 1.550 & 0.8511 & 0.04 & 1.950 & 1.6421 & 0.05 & 2.525 & 2.4630 & 0.06 & 3.150 & 3.2788 \\
\hline 0.7 & $\{0.3,0.2,0.1\}$ & 0.04 & 1.175 & 0.7604 & 0.07 & 1.675 & 1.4675 & 0.10 & 2.300 & 2.1557 & 0.12 & 2.875 & 2.8862 \\
\hline 0.6 & $\{0.3,0.2,0.1\}$ & 0.07 & 0.900 & 0.6544 & 0.12 & 1.500 & 1.2494 & 0.16 & 2.075 & 1.8776 & 0.19 & 2.600 & 2.5352 \\
\hline$\rho_{1}$ & $\rho_{2}$ & \multicolumn{12}{|c|}{$A R L_{0}=370$} \\
\hline 0.9 & $\{0.3,0.2,0.1\}$ & 0.01 & 2.450 & 0.8898 & 0.01 & 3.225 & 1.8431 & 0.01 & 3.650 & 2.8097 & 0.02 & 4.100 & 3.6211 \\
\hline 0.8 & $\{0.3,0.2,0.1\}$ & 0.02 & 2.025 & 0.8201 & 0.03 & 2.425 & 1.6595 & 0.04 & 2.675 & 2.4891 & 0.05 & 3.250 & 3.2935 \\
\hline 0.7 & $\{0.3,0.2,0.1\}$ & 0.04 & 1.550 & 0.7222 & 0.06 & 1.750 & 1.4753 & 0.08 & 2.350 & 2.1926 & 0.10 & 2.925 & 2.9083 \\
\hline 0.6 & $\{0.3,0.2,0.1\}$ & 0.06 & 1.050 & 0.6567 & 0.10 & 1.550 & 1.2727 & 0.14 & 2.100 & 1.8780 & 0.17 & 2.650 & 2.5264 \\
\hline$\rho_{1}$ & $\rho_{2}$ & \multicolumn{12}{|c|}{$A R L_{0}=500$} \\
\hline 0.9 & $\{0.3,0.2,0.1\}$ & 0.01 & 2.725 & 0.8787 & 0.01 & 3.575 & 1.8267 & 0.01 & 4.050 & 2.7891 & 0.02 & 4.475 & 3.5906 \\
\hline 0.8 & $\{0.3,0.2,0.1\}$ & 0.02 & 2.250 & 0.8058 & 0.03 & 2.675 & 1.6356 & 0.04 & 2.900 & 2.4597 & 0.05 & 3.300 & 3.2644 \\
\hline 0.7 & $\{0.3,0.2,0.1\}$ & 0.03 & 1.650 & 0.7559 & 0.05 & 1.825 & 1.5135 & 0.07 & 2.375 & 2.2226 & 0.09 & 2.975 & 2.9367 \\
\hline 0.6 & $\{0.3,0.2,0.1\}$ & 0.06 & 1.175 & 0.6386 & 0.09 & 1.575 & 1.2912 & 0.13 & 2.125 & 1.8888 & 0.16 & 2.675 & 2.5303 \\
\hline
\end{tabular}




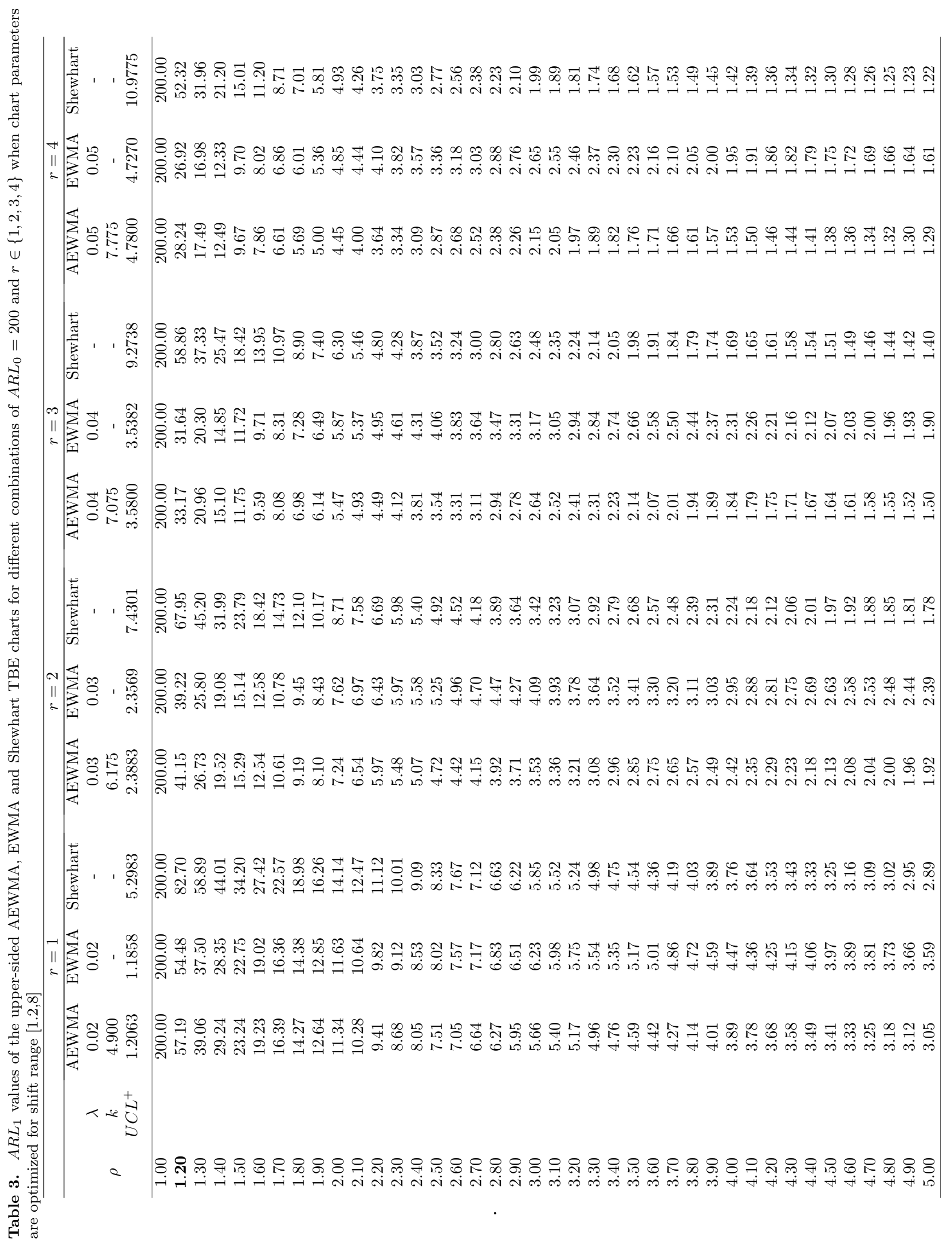




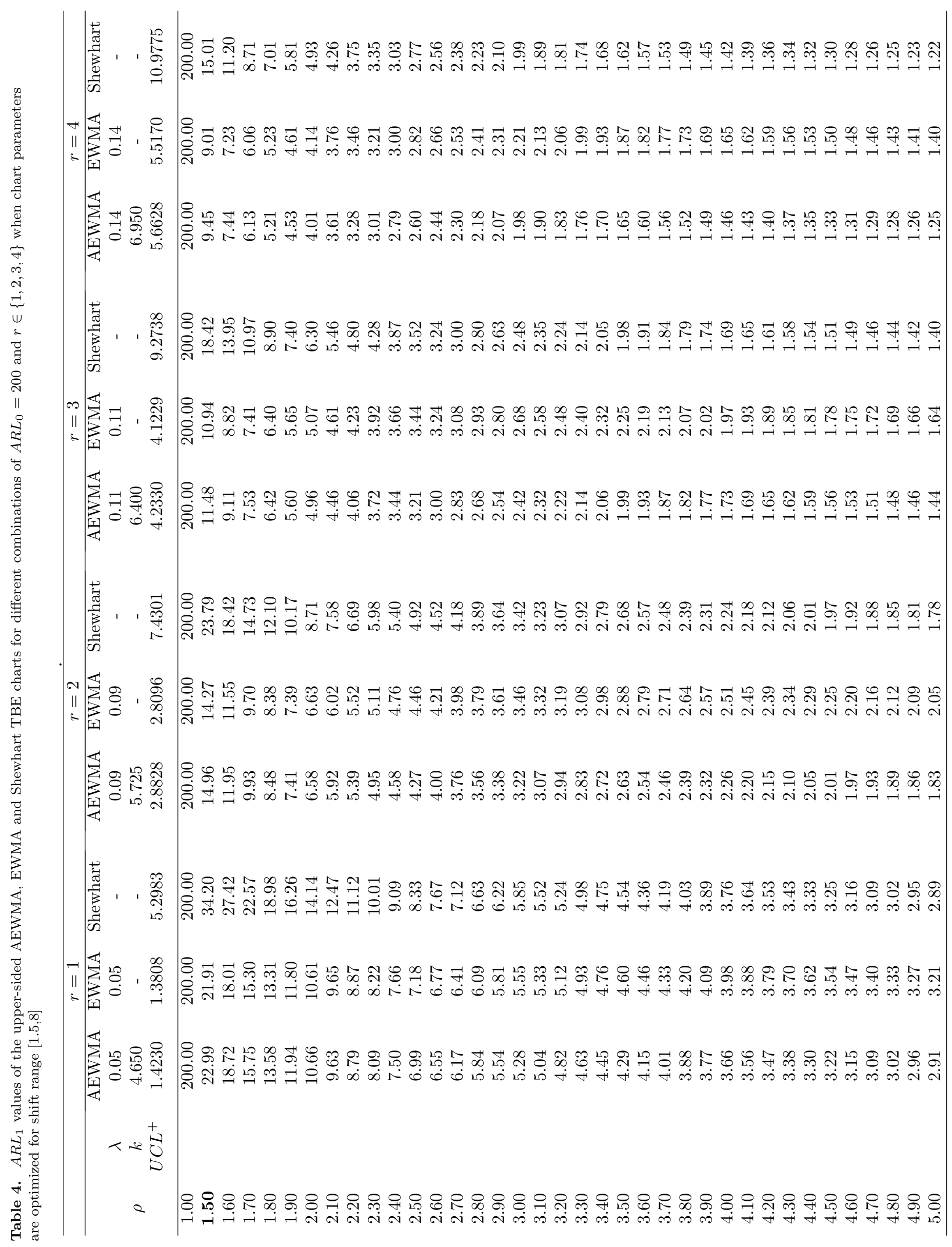




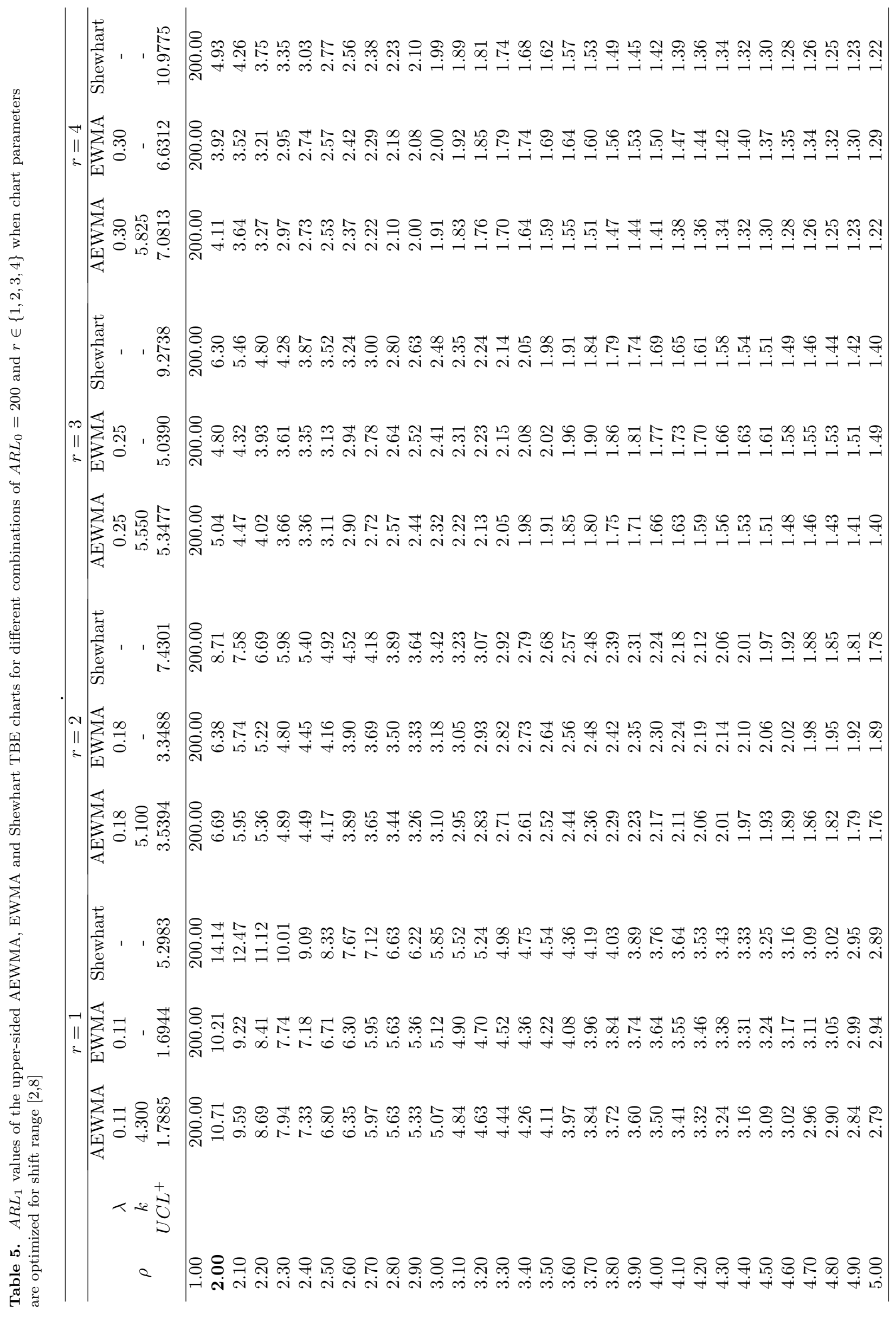




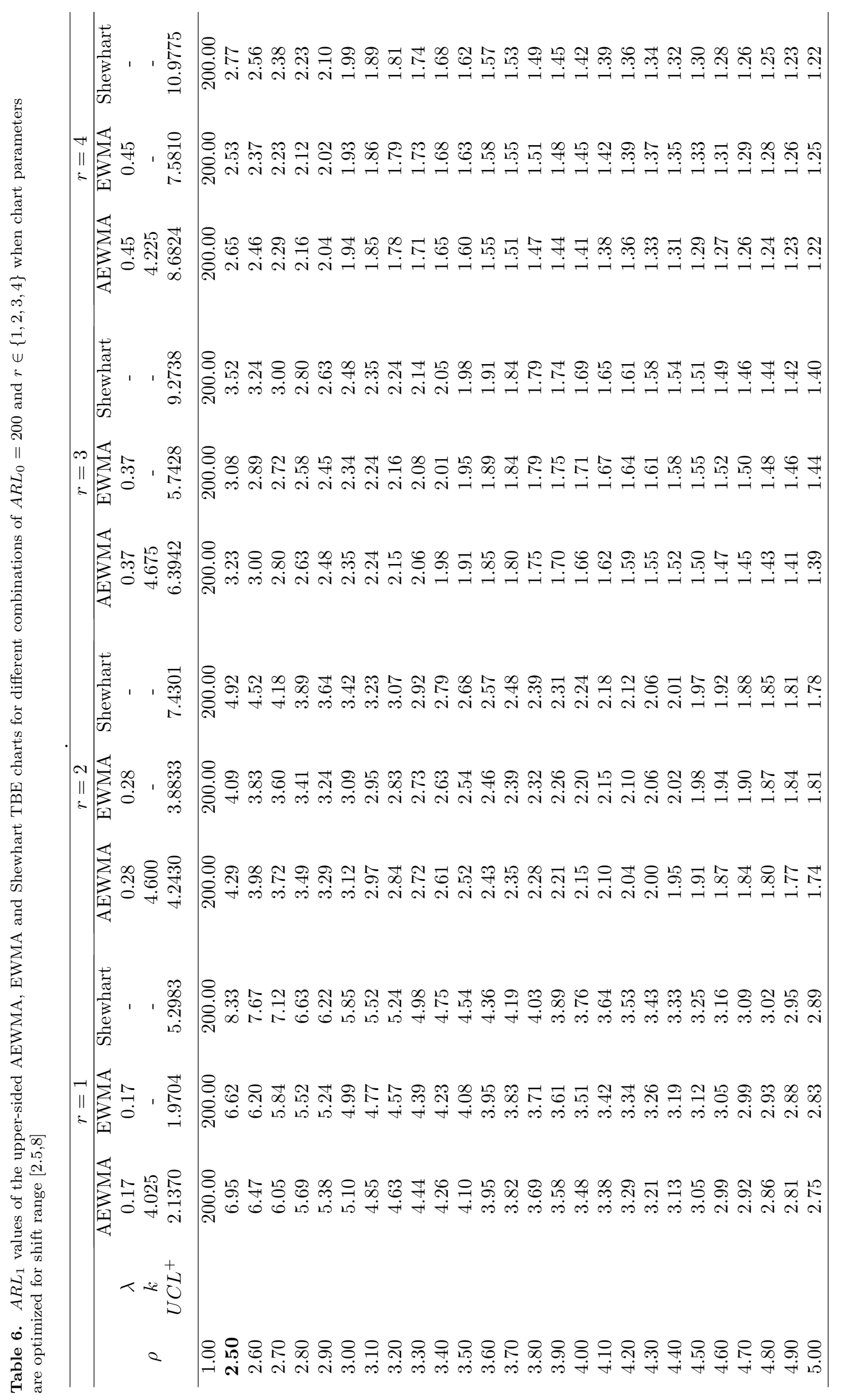




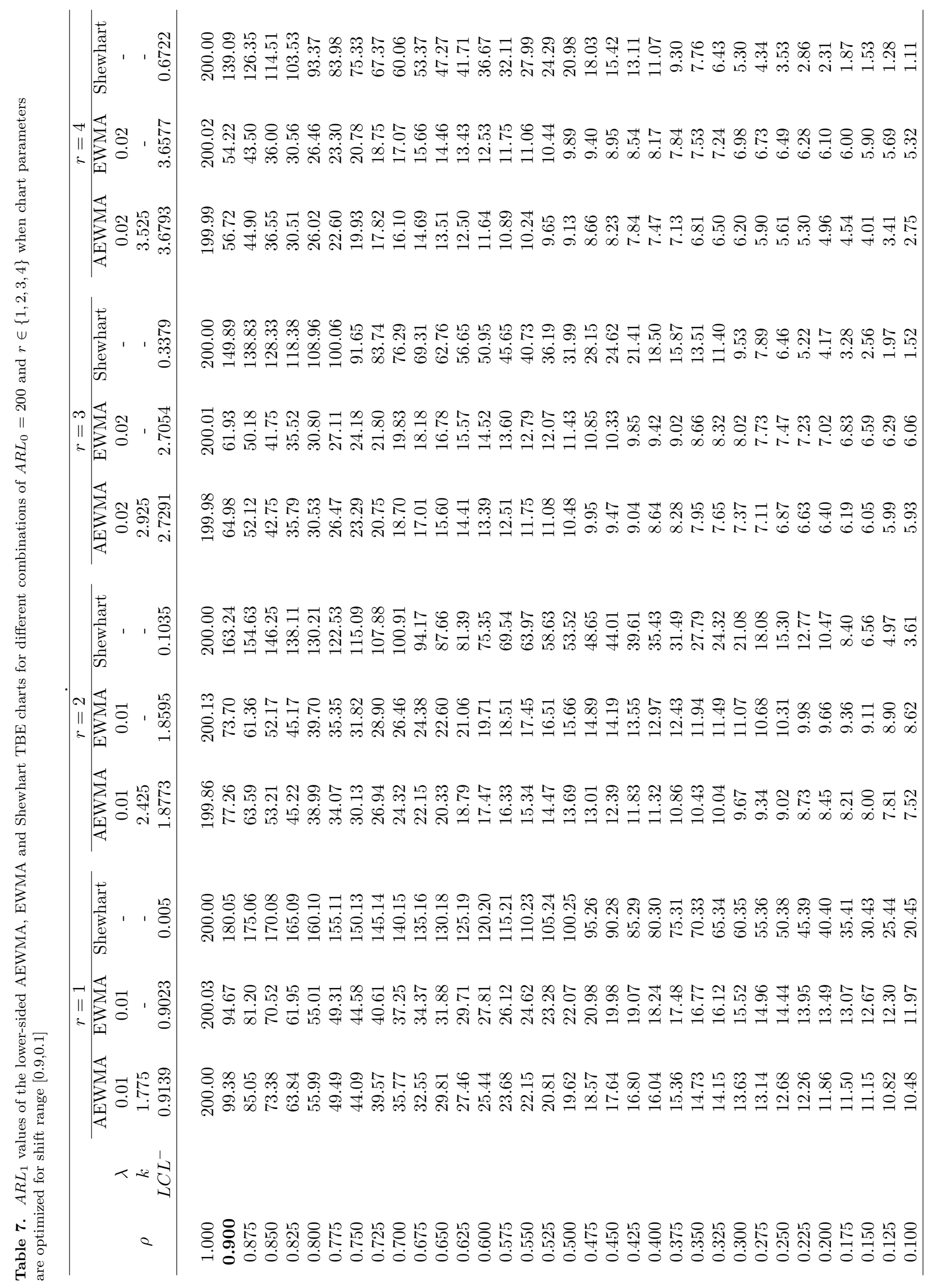




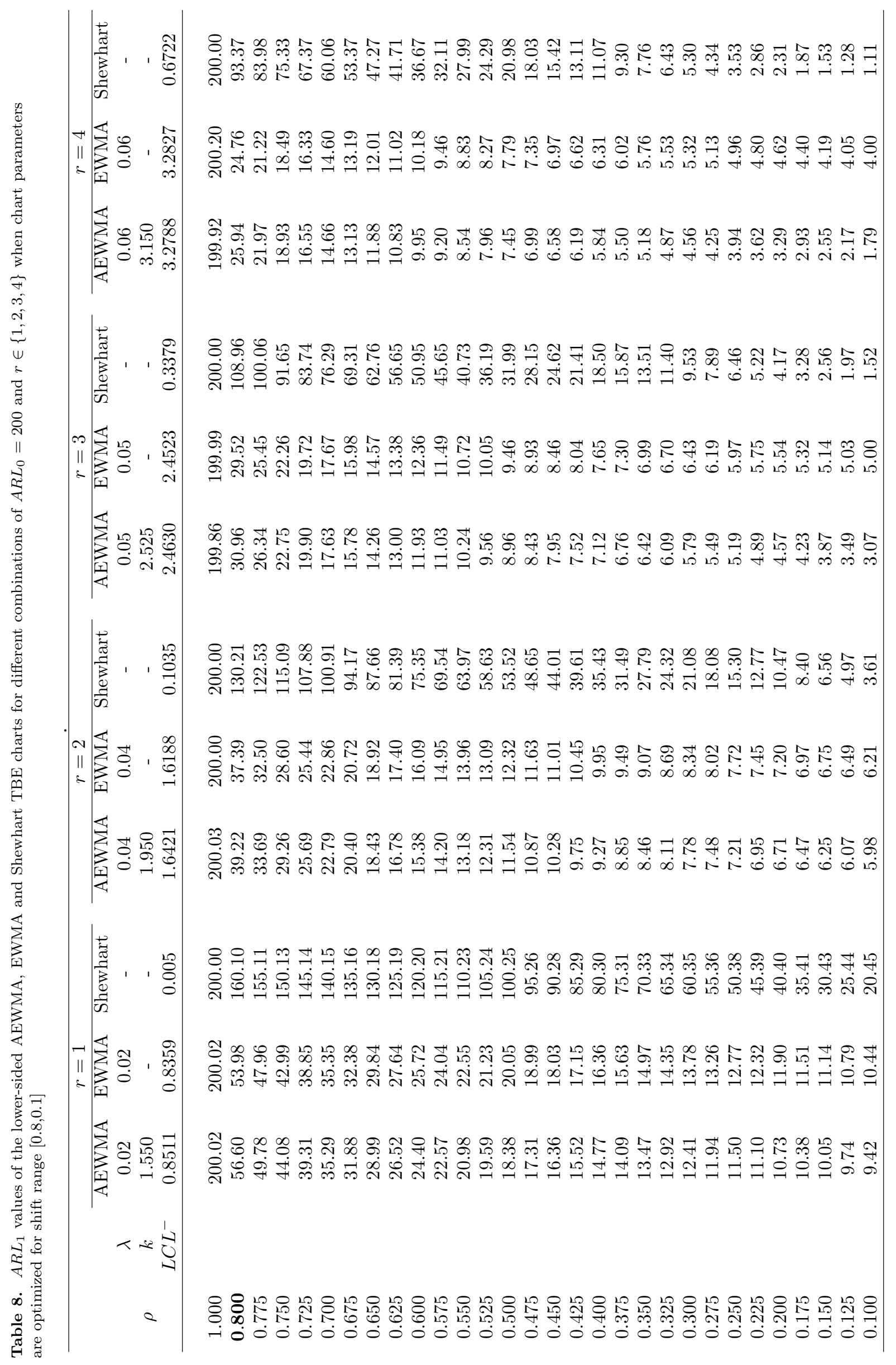




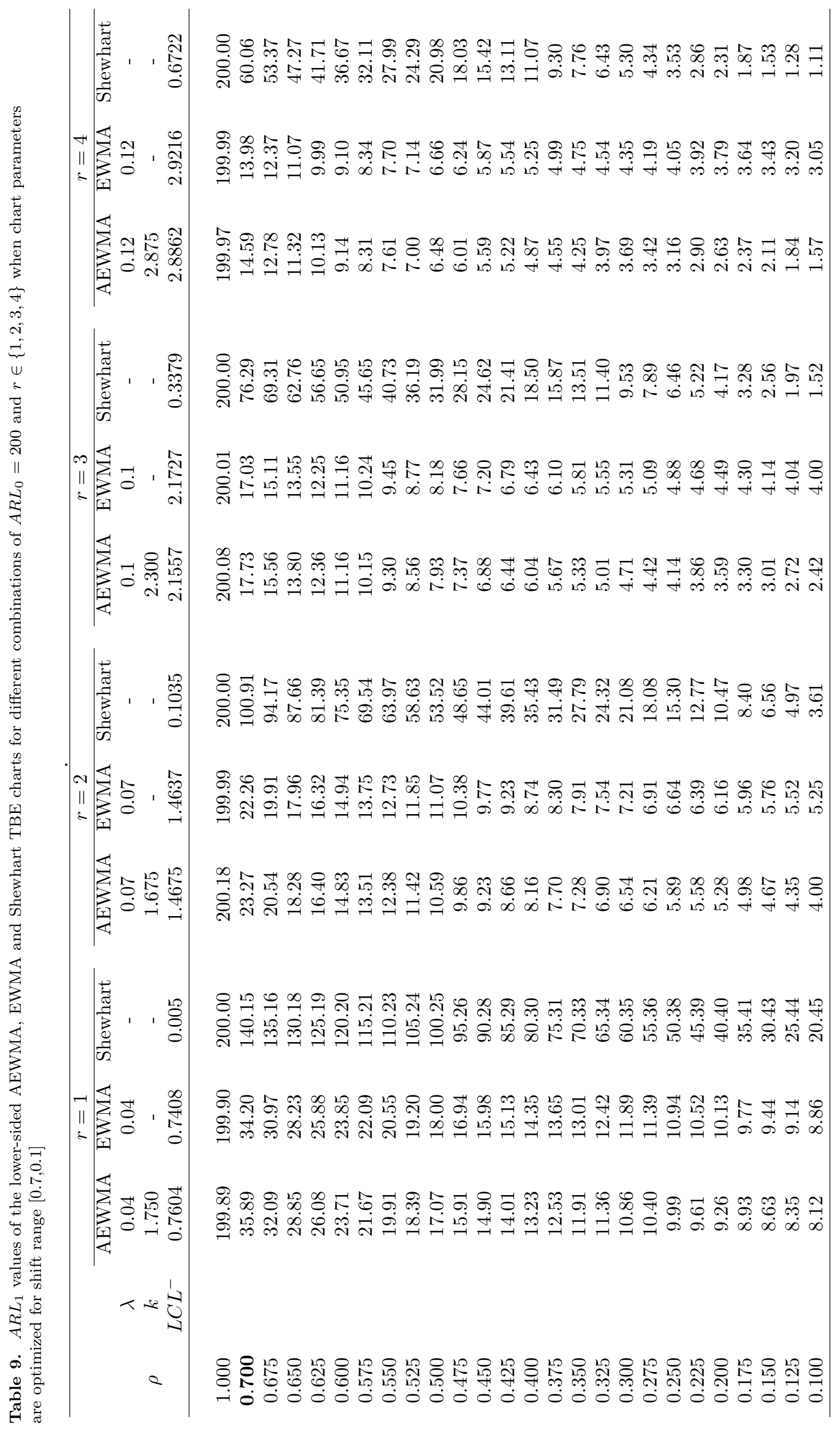




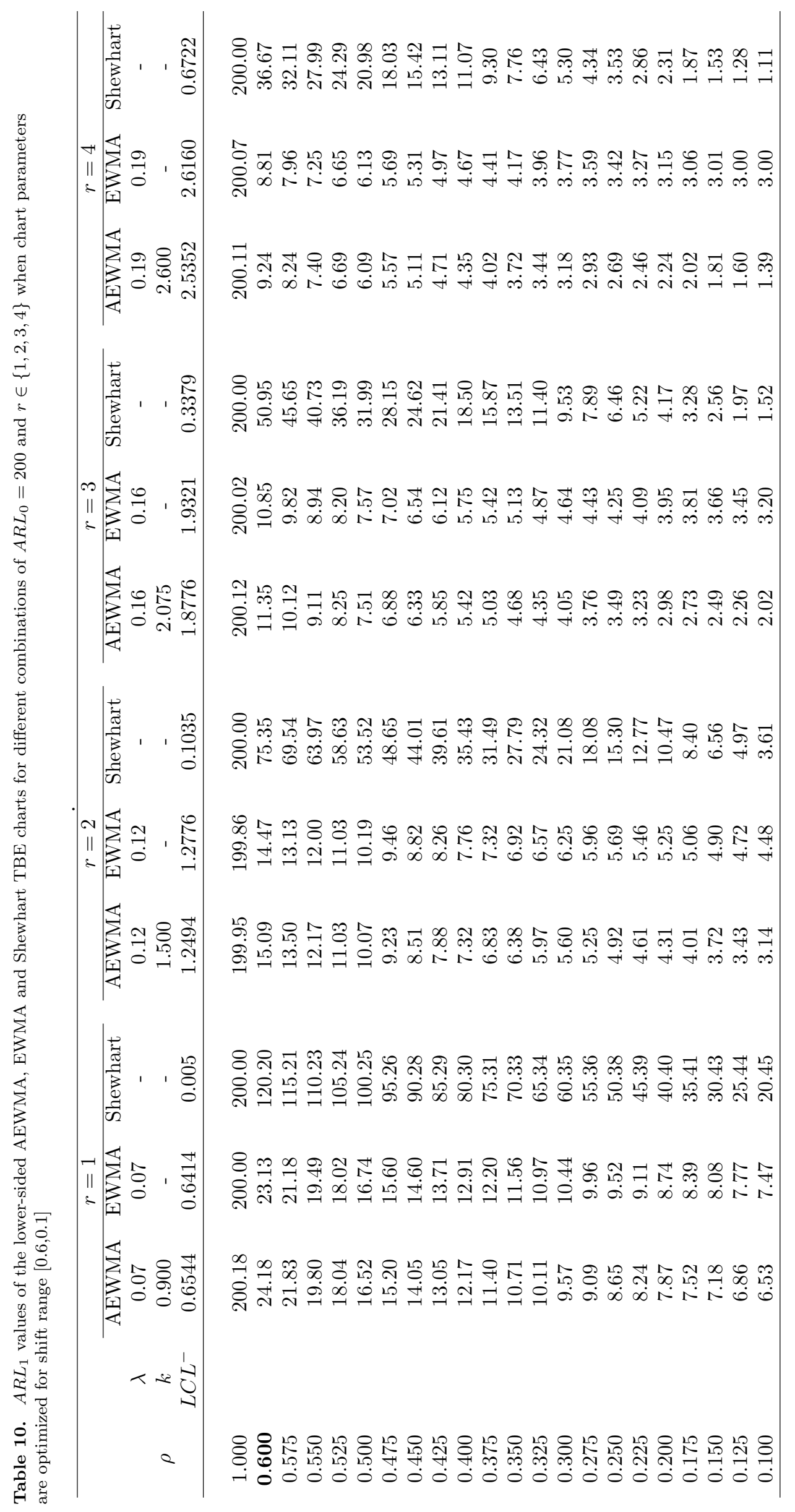




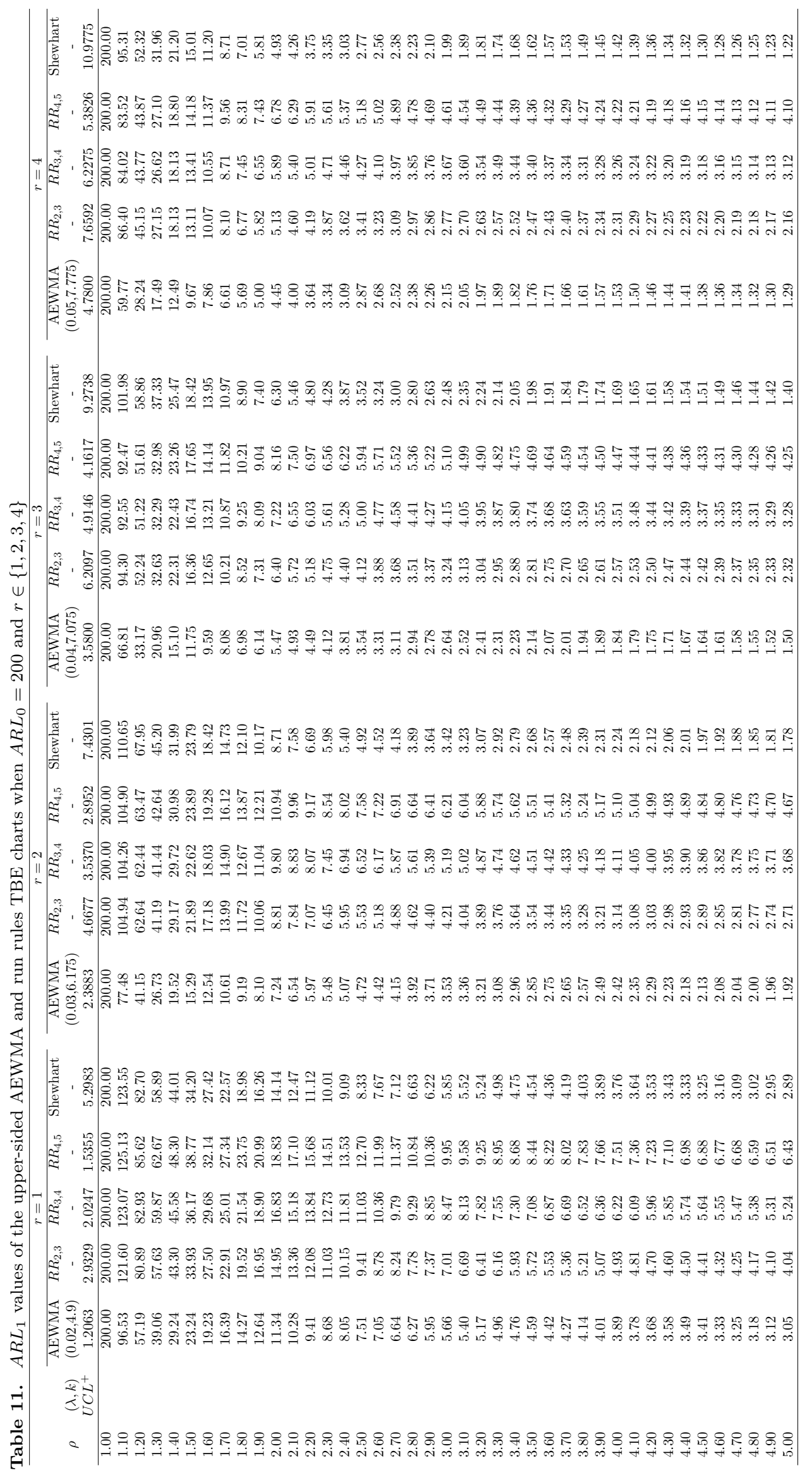




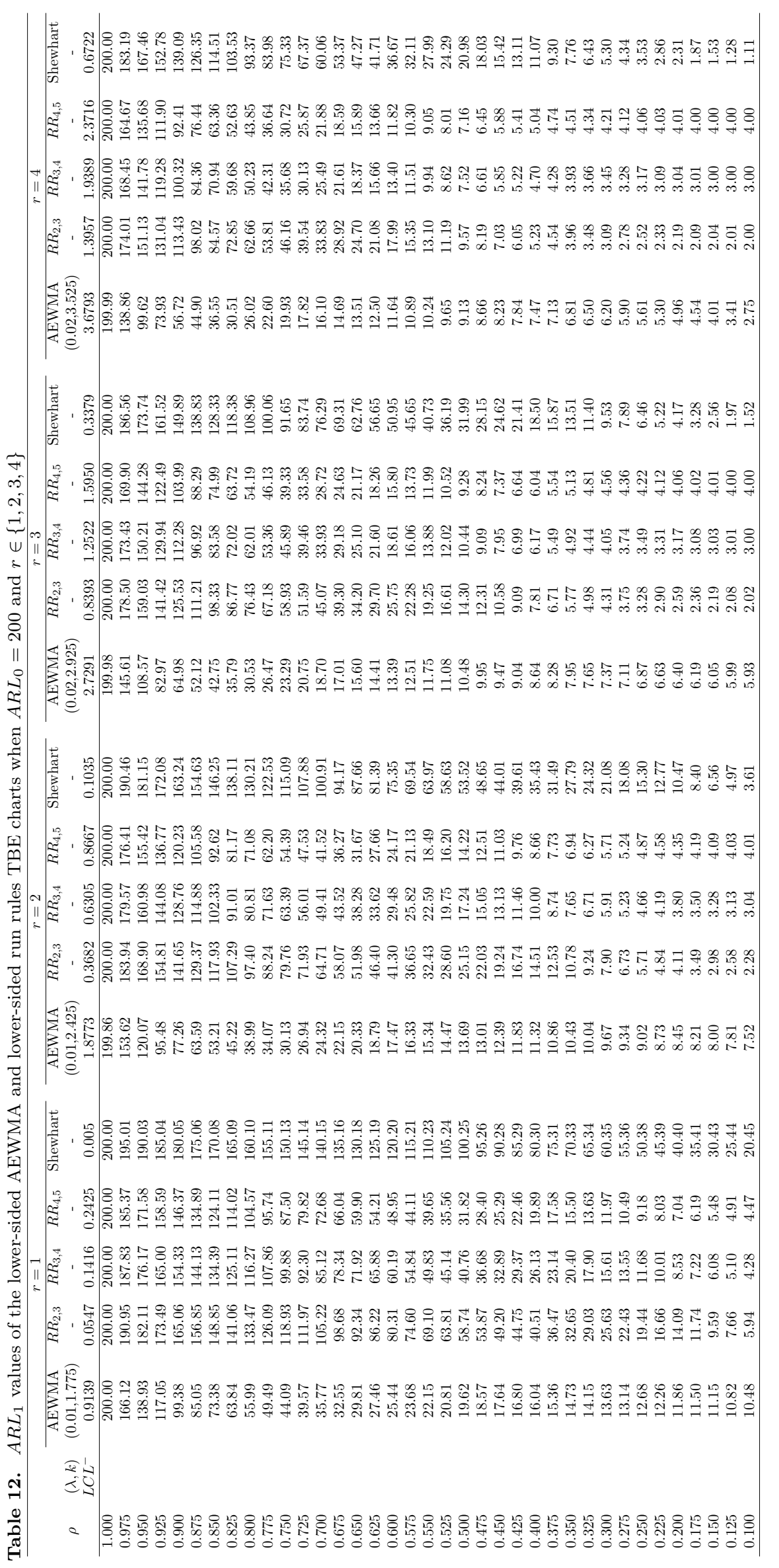


Table 13. $A R L_{1}$ values of the upper-sided AEWMA and DEWMA TBE charts when $A R L_{0}=200$ and $r \in\{1,2,3,4\}$

\begin{tabular}{|c|c|c|c|c|c|c|c|c|c|}
\hline \multirow{5}{*}{$\rho$} & & \multicolumn{2}{|c|}{$r=1$} & \multicolumn{2}{|c|}{$r=2$} & \multicolumn{2}{|c|}{$r=3$} & \multicolumn{2}{|c|}{$r=4$} \\
\hline & & AEWMA & DEWMA & AEWMA & DEWMA & AEWMA & DEWMA & AEWMA & DEWMA \\
\hline & $\lambda$ & 0.02 & 0.02 & 0.03 & 0.03 & 0.04 & 0.04 & 0.05 & 0.05 \\
\hline & $k$ & 4.900 & - & 6.175 & - & 7.075 & - & 7.775 & - \\
\hline & $U C L^{+}$ & 1.2063 & 1.0250 & 2.3883 & 2.0781 & 3.5800 & 3.1482 & 4.7800 & 4.2300 \\
\hline 1.00 & & 200.00 & 200.38 & 200.00 & 200.09 & 200.00 & 200.01 & 200.00 & 200.18 \\
\hline 1.10 & & 96.50 & 72.56 & 77.47 & 60.34 & 66.82 & 52.47 & 59.76 & 47.14 \\
\hline 1.20 & & 57.17 & 42.98 & 41.14 & 33.96 & 33.18 & 28.54 & 28.24 & 24.93 \\
\hline 1.30 & & 39.05 & 30.98 & 26.73 & 24.53 & 20.96 & 20.57 & 17.49 & 17.76 \\
\hline 1.40 & & 29.24 & 24.83 & 19.52 & 19.75 & 15.11 & 16.58 & 12.49 & 14.28 \\
\hline 1.50 & & 23.23 & 21.21 & 15.29 & 16.94 & 11.75 & 14.17 & 9.67 & 12.25 \\
\hline 1.60 & & 19.23 & 18.72 & 12.54 & 14.98 & 9.59 & 12.54 & 7.86 & 10.85 \\
\hline 1.70 & & 16.39 & 16.89 & 10.61 & 13.60 & 8.08 & 11.39 & 6.61 & 9.82 \\
\hline 1.80 & & 14.27 & 15.47 & 9.19 & 12.50 & 6.98 & 10.49 & 5.69 & 9.04 \\
\hline 1.90 & & 12.64 & 14.39 & 8.10 & 11.64 & 6.14 & 9.76 & 5.00 & 8.42 \\
\hline 2.00 & & 11.34 & 13.50 & 7.24 & 10.93 & 5.47 & 9.17 & 4.45 & 7.89 \\
\hline 2.10 & & 10.28 & 12.76 & 6.54 & 10.30 & 4.93 & 8.66 & 4.00 & 7.47 \\
\hline 2.20 & & 9.41 & 12.12 & 5.97 & 9.82 & 4.49 & 8.25 & 3.64 & 7.10 \\
\hline 2.30 & & 8.68 & 11.60 & 5.48 & 9.38 & 4.12 & 7.87 & 3.34 & 6.78 \\
\hline 2.40 & & 8.05 & 11.09 & 5.07 & 9.00 & 3.81 & 7.55 & 3.09 & 6.50 \\
\hline 2.50 & & 7.51 & 10.65 & 4.72 & 8.66 & 3.54 & 7.25 & 2.87 & 6.25 \\
\hline 2.60 & & 7.04 & 10.29 & 4.42 & 8.36 & 3.31 & 7.01 & 2.68 & 6.02 \\
\hline 2.70 & & 6.63 & 9.96 & 4.15 & 8.08 & 3.11 & 6.77 & 2.52 & 5.82 \\
\hline 2.80 & & 6.27 & 9.63 & 3.92 & 7.81 & 2.94 & 6.56 & 2.38 & 5.65 \\
\hline 2.90 & & 5.95 & 9.35 & 3.71 & 7.59 & 2.78 & 6.36 & 2.26 & 5.48 \\
\hline 3.00 & & 5.66 & 9.09 & 3.53 & 7.39 & 2.64 & 6.19 & 2.15 & 5.33 \\
\hline 3.10 & & 5.40 & 8.86 & 3.36 & 7.19 & 2.52 & 6.02 & 2.05 & 5.19 \\
\hline 3.20 & & 5.17 & 8.63 & 3.21 & 7.01 & 2.41 & 5.88 & 1.97 & 5.05 \\
\hline 3.30 & & 4.96 & 8.42 & 3.08 & 6.85 & 2.31 & 5.73 & 1.89 & 4.94 \\
\hline 3.40 & & 4.76 & 8.25 & 2.96 & 6.70 & 2.23 & 5.61 & 1.82 & 4.82 \\
\hline 3.50 & & 4.59 & 8.06 & 2.85 & 6.55 & 2.14 & 5.49 & 1.76 & 4.72 \\
\hline 3.60 & & 4.42 & 7.90 & 2.75 & 6.41 & 2.07 & 5.37 & 1.71 & 4.62 \\
\hline 3.70 & & 4.27 & 7.74 & 2.65 & 6.28 & 2.01 & 5.26 & 1.66 & 4.53 \\
\hline 3.80 & & 4.14 & 7.58 & 2.57 & 6.17 & 1.94 & 5.16 & 1.61 & 4.44 \\
\hline 3.90 & & 4.01 & 7.46 & 2.49 & 6.05 & 1.89 & 5.07 & 1.57 & 4.36 \\
\hline 4.00 & & 3.89 & 7.32 & 2.42 & 5.95 & 1.84 & 4.98 & 1.53 & 4.28 \\
\hline 4.10 & & 3.78 & 7.21 & 2.35 & 5.84 & 1.79 & 4.89 & 1.50 & 4.21 \\
\hline 4.20 & & 3.68 & 7.09 & 2.29 & 5.74 & 1.75 & 4.80 & 1.46 & 4.14 \\
\hline 4.30 & & 3.58 & 6.97 & 2.23 & 5.65 & 1.71 & 4.73 & 1.44 & 4.07 \\
\hline 4.40 & & 3.49 & 6.86 & 2.18 & 5.56 & 1.67 & 4.66 & 1.41 & 4.00 \\
\hline 4.50 & & 3.41 & 6.76 & 2.13 & 5.49 & 1.64 & 4.59 & 1.38 & 3.95 \\
\hline 4.60 & & 3.33 & 6.67 & 2.08 & 5.40 & 1.61 & 4.52 & 1.36 & 3.89 \\
\hline 4.70 & & 3.25 & 6.57 & 2.04 & 5.32 & 1.58 & 4.46 & 1.34 & 3.84 \\
\hline 4.80 & & 3.18 & 6.48 & 2.00 & 5.26 & 1.55 & 4.40 & 1.32 & 3.78 \\
\hline 4.90 & & 3.12 & 6.39 & 1.96 & 5.18 & 1.52 & 4.34 & 1.30 & 3.74 \\
\hline 5.00 & & 3.05 & 6.32 & 1.92 & 5.12 & 1.50 & 4.28 & 1.29 & 3.68 \\
\hline
\end{tabular}


Table 14. $A R L_{1}$ values of the lower-sided AEWMA and DEWMA TBE charts when $A R L_{0}=200$ and $r \in\{1,2,3,4\}$

\begin{tabular}{|c|c|c|c|c|c|c|c|c|c|}
\hline \multirow{5}{*}{$\rho$} & & \multicolumn{2}{|c|}{$r=1$} & \multicolumn{2}{|c|}{$r=2$} & \multicolumn{2}{|c|}{$r=3$} & \multicolumn{2}{|c|}{$r=4$} \\
\hline & & AEWMA & DEWMA & AEWMA & DEWMA & AEWMA & DEWMA & AEWMA & DEWMA \\
\hline & $\lambda$ & 0.01 & 0.01 & 0.01 & 0.01 & 0.02 & 0.02 & 0.02 & 0.02 \\
\hline & $k$ & 1.775 & - & 2.425 & - & 2.925 & - & 3.525 & - \\
\hline & $L C L^{-}$ & 0.9139 & 0.9950 & 1.8773 & 1.9934 & 2.7291 & 2.9499 & 3.6793 & 3.9425 \\
\hline 1.00 & & 199.84 & 202.24 & 199.63 & 201.29 & 199.97 & 200.78 & 200.04 & 199.99 \\
\hline 0.975 & & 165.99 & 134.07 & 153.46 & 113.70 & 145.60 & 117.03 & 138.89 & 109.90 \\
\hline 0.950 & & 138.83 & 94.66 & 119.95 & 74.48 & 108.56 & 79.34 & 99.64 & 71.46 \\
\hline 0.925 & & 116.97 & 71.41 & 95.40 & 53.57 & 82.97 & 58.03 & 73.95 & 51.70 \\
\hline 0.900 & & 99.32 & 56.27 & 77.20 & 41.33 & 64.98 & 45.67 & 56.73 & 40.60 \\
\hline 0.875 & & 85.00 & 46.33 & 63.55 & 33.81 & 52.12 & 37.67 & 44.90 & 33.61 \\
\hline 0.850 & & 73.35 & 39.08 & 53.18 & 28.72 & 42.75 & 32.39 & 36.56 & 28.98 \\
\hline 0.825 & & 63.81 & 34.00 & 45.20 & 25.18 & 35.79 & 28.66 & 30.51 & 25.66 \\
\hline 0.800 & & 55.96 & 30.19 & 38.97 & 22.46 & 30.53 & 25.88 & 26.02 & 23.28 \\
\hline 0.775 & & 49.47 & 27.19 & 34.05 & 20.43 & 26.47 & 23.69 & 22.60 & 21.45 \\
\hline 0.750 & & 44.07 & 24.90 & 30.12 & 18.92 & 23.29 & 21.97 & 19.93 & 19.93 \\
\hline 0.725 & & 39.56 & 23.00 & 26.93 & 17.61 & 20.75 & 20.55 & 17.82 & 18.71 \\
\hline 0.700 & & 35.76 & 21.46 & 24.31 & 16.59 & 18.70 & 19.40 & 16.10 & 17.72 \\
\hline 0.675 & & 32.54 & 20.21 & 22.14 & 15.72 & 17.01 & 18.43 & 14.69 & 16.84 \\
\hline 0.650 & & 29.80 & 19.13 & 20.32 & 14.98 & 15.60 & 17.57 & 13.51 & 16.09 \\
\hline 0.625 & & 27.45 & 18.25 & 18.78 & 14.32 & 14.41 & 16.84 & 12.50 & 15.41 \\
\hline 0.600 & & 25.43 & 17.44 & 17.46 & 13.78 & 13.39 & 16.18 & 11.64 & 14.85 \\
\hline 0.575 & & 23.67 & 16.74 & 16.32 & 13.28 & 12.51 & 15.60 & 10.89 & 14.32 \\
\hline 0.550 & & 22.14 & 16.15 & 15.33 & 12.85 & 11.75 & 15.08 & 10.24 & 13.85 \\
\hline 0.525 & & 20.80 & 15.60 & 14.46 & 12.43 & 11.08 & 14.61 & 9.65 & 13.43 \\
\hline 0.500 & & 19.62 & 15.10 & 13.69 & 12.08 & 10.48 & 14.17 & 9.13 & 13.04 \\
\hline 0.475 & & 18.57 & 14.66 & 13.00 & 11.74 & 9.95 & 13.78 & 8.66 & 12.68 \\
\hline 0.450 & & 17.63 & 14.26 & 12.38 & 11.44 & 9.47 & 13.41 & 8.23 & 12.35 \\
\hline 0.425 & & 16.79 & 13.89 & 11.83 & 11.15 & 9.04 & 13.08 & 7.84 & 12.05 \\
\hline 0.400 & & 16.04 & 13.56 & 11.32 & 10.90 & 8.64 & 12.77 & 7.47 & 11.76 \\
\hline 0.375 & & 15.35 & 13.24 & 10.85 & 10.66 & 8.28 & 12.49 & 7.13 & 11.50 \\
\hline 0.350 & & 14.73 & 12.95 & 10.43 & 10.43 & 7.95 & 12.21 & 6.81 & 11.26 \\
\hline 0.325 & & 14.15 & 12.67 & 10.03 & 10.23 & 7.65 & 11.96 & 6.50 & 11.03 \\
\hline 0.300 & & 13.62 & 12.42 & 9.67 & 10.03 & 7.37 & 11.71 & 6.20 & 10.82 \\
\hline 0.275 & & 13.13 & 12.18 & 9.33 & 9.84 & 7.11 & 11.47 & 5.90 & 10.59 \\
\hline 0.250 & & 12.68 & 11.95 & 9.02 & 9.65 & 6.87 & 11.26 & 5.61 & 10.36 \\
\hline 0.225 & & 12.26 & 11.74 & 8.72 & 9.45 & 6.63 & 11.09 & 5.30 & 10.16 \\
\hline 0.200 & & 11.86 & 11.53 & 8.45 & 9.28 & 6.40 & 10.96 & 4.96 & 10.04 \\
\hline 0.175 & & 11.49 & 11.34 & 8.21 & 9.13 & 6.19 & 10.81 & 4.54 & 9.99 \\
\hline 0.150 & & 11.15 & 11.17 & 8.00 & 9.04 & 6.05 & 10.54 & 4.01 & 9.93 \\
\hline 0.125 & & 10.82 & 11.06 & 7.81 & 9.00 & 5.99 & 10.21 & 3.41 & 9.71 \\
\hline 0.100 & & 10.48 & 10.99 & 7.51 & 9.00 & 5.93 & 10.02 & 2.75 & 9.25 \\
\hline
\end{tabular}

Table 15. Time between consecutive accidents of F-16 from December 1 of 1988 to December 31 of 2017

\begin{tabular}{cccc}
\hline No. & $X($ Days $)$ & No. & $X($ Days $)$ \\
\hline 1 & 1456 & 9 & 499 \\
2 & 231 & 10 & 587 \\
3 & 691 & 11 & 561 \\
4 & 122 & 12 & 547 \\
5 & 718 & 13 & 448 \\
6 & 1147 & 14 & 1561 \\
7 & 225 & 15 & 53 \\
8 & 706 & 16 & 280 \\
\hline
\end{tabular}


Table 16. The scaled dataset and the corresponding values of the monitoring statistics of different TBE charts

\begin{tabular}{ccccc}
\hline & & AEWMA & EWMA & DEWMA \\
No. & $X / 1500$ & $Z_{t}^{-}$ & $Z_{t}^{-}$ & $Z_{t}$ \\
\hline 1 & 0.9707 & 0.9979 & 0.9979 & 0.9999 \\
2 & 0.1540 & 0.9389 & 0.9389 & 0.9956 \\
3 & 0.4607 & 0.9054 & 0.9054 & 0.9893 \\
4 & 0.0813 & 0.8477 & 0.8477 & 0.9794 \\
5 & 0.4787 & 0.8219 & 0.8219 & 0.9683 \\
6 & 0.7647 & 0.8179 & 0.8179 & 0.9578 \\
7 & 0.1500 & 0.7711 & 0.7711 & 0.9447 \\
8 & 0.4707 & 0.7501 & 0.7501 & 0.9311 \\
9 & 0.3327 & 0.7209 & 0.7209 & 0.9164 \\
10 & 0.3913 & 0.6978 & 0.6978 & 0.9011 \\
11 & 0.3740 & 0.6751 & 0.6751 & 0.8853 \\
12 & 0.3647 & $\mathbf{0 . 6 5 3 4}$ & 0.6534 & 0.8690 \\
13 & 0.2987 & 0.6286 & $\mathbf{0 . 6 2 8 6}$ & 0.8522 \\
14 & 1.0407 & 0.6574 & 0.6574 & 0.8386 \\
15 & 0.0353 & 0.6139 & 0.6139 & $\mathbf{0 . 8 2 2 8}$ \\
16 & 0.1867 & 0.5840 & 0.5840 & 0.8061 \\
\hline
\end{tabular}




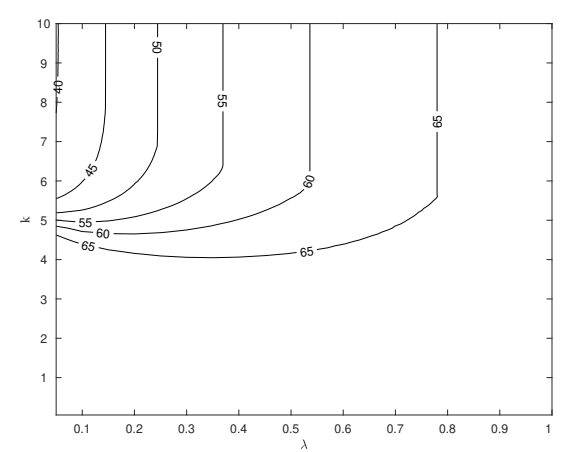

(a) $\rho=1.2$

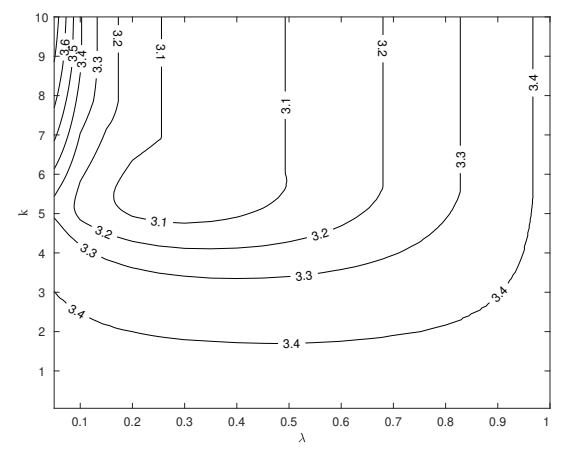

(e) $\rho=3$

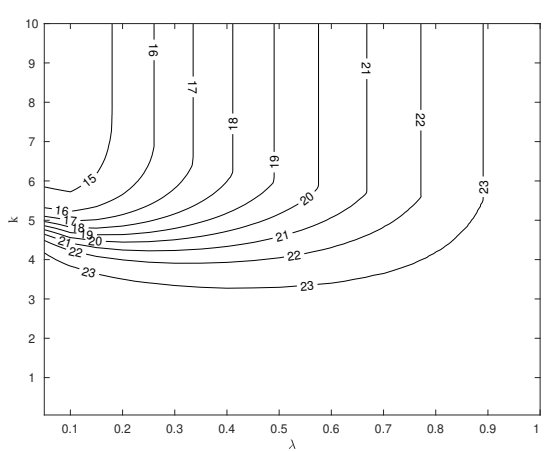

(c) $\rho=1.5$

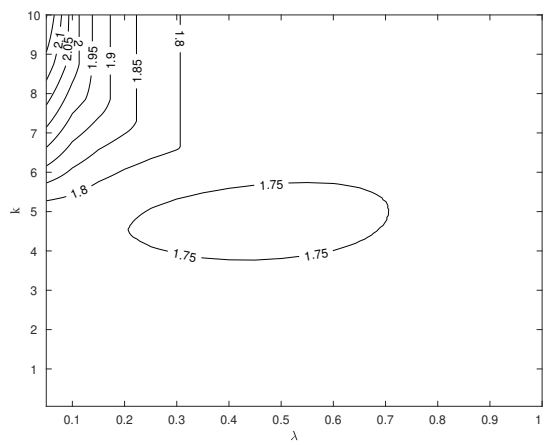

(h) $\rho=5$

Figure 1. Contour plots of the $A R L_{1}$ values of the upper-sided AEWMA chart for Gamma TBE data when $A R L_{0}=200$ and $r=2$. 


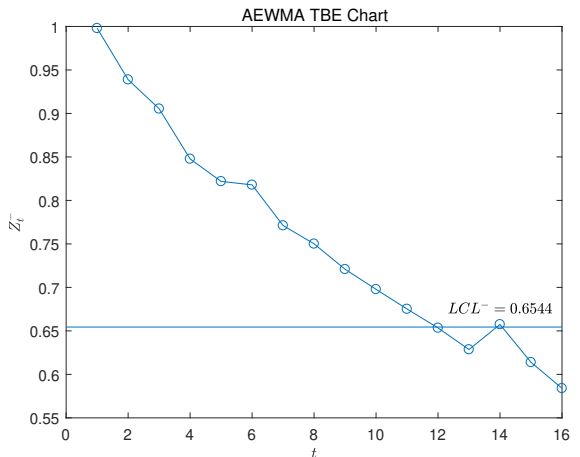

(a) AEWMA TBE chart

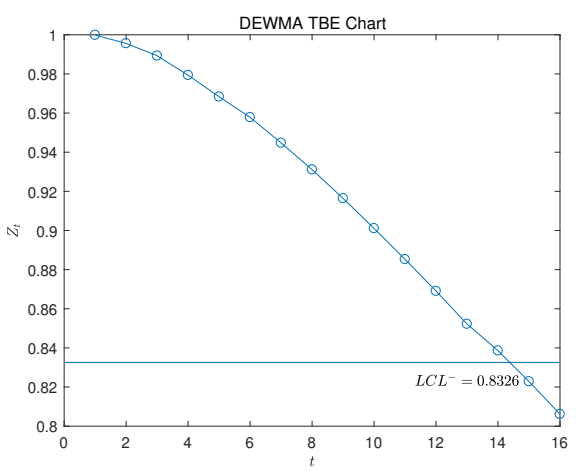

(c) DEWMA TBE chart

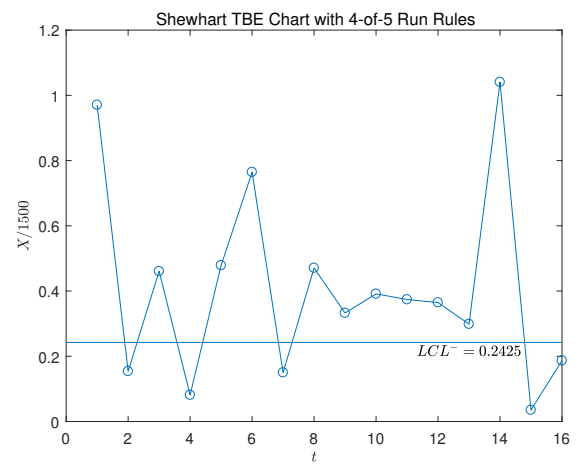

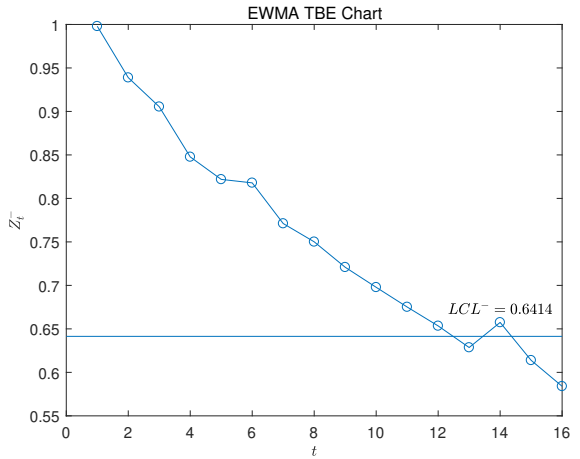

(b) EWMA TBE chart

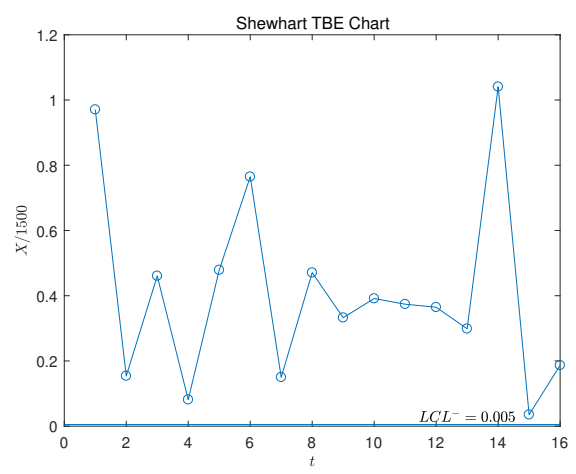

(d) Shewhart TBE chart

(e) Shewhart TBE chart with 4-of-5 Run Rules

Figure 2. Lower-sided TBE charts for the scaled TBE dataset in Table 16 